\title{
Existence and approximation for Navier-Stokes system with Tresca's friction at the boundary and heat transfer governed by Cattaneo's law
}

\author{
Special Issue Inequality Problems in Contact Mechanics \\ Mahdi Boukrouche', Imane Boussetouan ${ }^{2}$ and Laetitia Paoli ${ }^{1}$
}

\begin{abstract}
We consider an unsteady non-isothermal incompressible fluid flow. We model heat conduction with Cattaneo's law instead of the commonly used Fourier's law in order to overcome the physical paradox of infinite propagation speed. We assume that the fluid viscosity depends on the temperature while the thermal capacity depends on the velocity field. The problem is thus described by Navier-Stokes system coupled with the hyperbolic heat equation. Furthermore we consider non-standard boundary conditions with Tresca's friction law on a part of the boundary. By using a time-splitting technique, we construct a sequence of decoupled approximate problems and we prove the convergence of the corresponding approximate solutions, leading to an existence theorem for the coupled fluid flow/heat transfer problem. Finally we present some numerical results.
\end{abstract}

\section{Keywords}

Navier-Stokes system, hyperbolic heat equation, Tresca's friction law, time-splitting technique, convergence

\section{Introduction}

Many modern industrial processes, like lubrication or injection moulding for instance, involve nonstationary incompressible fluid flows and heat transfer. Such problems are described by the Navier-Stokes

\footnotetext{
${ }^{1}$ University of Lyon, UJM F-42023 Saint-Etienne, CNRS UMR 5208, Institut Camille Jordan, France

2 Ecole Préparatoire aux Sciences et Techniques d'Annaba, Annaba, Algeria
}

Corresponding author:

Laetitia Paoli, University of Lyon, UJM F-42023 Saint-Etienne, CNRS UMR 5208, Institut Camille Jordan, 23 rue Paul Michelon, 42023 Saint-Etienne Cedex 2, France.

Email: laetitia.paoli@univ-st-etienne.fr 
system

$$
\left\{\begin{array}{l}
\frac{\partial v}{\partial t}+(v \cdot \nabla) v=\operatorname{div}(\sigma)+f \\
\operatorname{div}(v)=0
\end{array}\right.
$$

where $f$ denotes the density of body forces, $v$ is the velocity field and $\sigma$ is the stress tensor given by

$$
\sigma=-p \operatorname{Id}+2 \mu D(v), \quad D(v)=\left(\frac{1}{2}\left(\frac{\partial v_{i}}{\partial x_{j}}+\frac{\partial v_{j}}{\partial x_{i}}\right)\right)_{1 \leq i, j \leq d}
$$

where Id is the identity matrix in $\mathbb{R}^{d}, \mu$ is the fluid viscosity and $p$ is the pressure, coupled with the heat transfer equation

$$
c \frac{\partial T}{\partial t}+\operatorname{div}(q)=\phi
$$

where $\phi$ is some dissipative term, $c$ is the thermal capacity of the fluid, $T$ is the temperature field and $q$ is the heat flux.

The latter is usually modeled by the Fourier's law $\left({ }^{1}\right)$ i.e.

$$
q=-K \nabla T
$$

where $K$ is the thermal conductivity of the media. By inserting (3) into (2), we obtain the parabolic equation

$$
c \frac{\partial T}{\partial t}-\operatorname{div}(K \nabla T)=\phi
$$

leading to infinite propagation speed. This property, known as the "heat paradox" does not fit with the observed behaviors, especially when temperature gradients or heat flux with short duration or high frequencies are applied $\left({ }^{2-11}\right)$. In order to get a more physically relevant description, several modifications of Fourier's law have been proposed $\left({ }^{12-15}\right)$. Among them, one of the most commonly adopted is due to Cattaneo $\left({ }^{16,17}\right)$ and consists in adding a damping term $d \frac{\partial^{2} T}{\partial t^{2}}$, with a positive parameter $d$, in the left hand side of (4) i.e

$$
d \frac{\partial^{2} T}{\partial t^{2}}+c \frac{\partial T}{\partial t}-\operatorname{div}(K \nabla T)=\phi .
$$

In the case of a constant thermal capacity and no dissipative term, equation (5) can be decomposed as

$$
\left\{\begin{array}{c}
c \frac{\partial T}{\partial t}+\operatorname{div}(q)=0 \\
q+\frac{d}{c} \frac{\partial q}{\partial t}=-K \nabla T
\end{array}\right.
$$

By identifying $q+\frac{d}{c} \frac{\partial q}{\partial t}$ to $q\left(\cdot+\frac{d}{c}\right)$ we obtain

$$
q\left(\cdot+\frac{d}{c}\right)=-K \nabla T
$$


and the ratio $d / c$ can be interpreted as the time-lag needed to establish steady-state heat conduction in a material element suddenly exposed to heat flux.

For this kind of fluid flow/heat transfer problem, a first study has been proposed in ${ }^{18}$ in the case of a 2D thin flow. By using an asymptotic expansion with respect to the thickness of the fluid domain, the authors obtain a decoupled system of equations for $(v, p)$ and $T$. In this paper, we do not introduce any restrictive assumption on the thickness of the 2D domain. We consider the coupled problem given by the Navier-Stokes system with a temperature-dependent viscosity $\left({ }^{19-23}\right)$ and the hyperbolic heat equation where the thermal capacity (which may vary under internal vibrations at the atomic scale ${ }^{19,24}$ ) and the dissipative term depend on the velocity field $\left({ }^{19}\right)$ i.e.

$$
(P)\left\{\begin{array}{l}
\frac{\partial v}{\partial t}+(v \cdot \nabla) v-2 \operatorname{div}(\mu(T) D(v))+\nabla p=f \\
\operatorname{div}(v)=0 \\
d \frac{\partial^{2} T}{\partial t^{2}}+c(v) \frac{\partial T}{\partial t}-\operatorname{div}(K \nabla T)=\phi(v)
\end{array}\right.
$$

with the initial conditions

$$
v(\cdot, 0)=v_{0}, T(\cdot, 0)=T_{0}, \frac{\partial T}{\partial t}(\cdot, 0)=T_{1} .
$$

Motivated by lubrication or extrusion/injection phenomena, we will consider also some non-standard boundary conditions for the fluid flow, namely non-homogeneous Dirichlet conditions on a part of the boundary and Tresca's friction law on the other part $\left({ }^{25-27}\right)$.

More precisely, let us define the domain $\Omega$ of the flow by

$$
\Omega=\left\{\left(x_{1}, x_{2}\right) \in \mathbb{R}^{2}: 0<x_{1}<L, 0<x_{2}<h\left(x_{1}\right)\right\},
$$

where $L>0$ and $h \in C^{1}(\mathbb{R} ; \mathbb{R})$ is bounded from above and from below by two positive real numbers. We decompose the boundary of $\Omega$ as $\partial \Omega=\Gamma_{0} \cup \Gamma_{L} \cup \Gamma_{1}$, with $\Gamma_{0}=\left\{\left(x_{1}, x_{2}\right) \in \bar{\Omega}: x_{2}=0\right\}, \Gamma_{1}=$ $\left\{\left(x_{1}, x_{2}\right) \in \bar{\Omega}: x_{2}=h\left(x_{1}\right)\right\}$ and $\Gamma_{L}$ the lateral boundary. We assume that the upper part of the boundary is fixed while the lower part is moving. We denote by $s: \Gamma_{0} \rightarrow \mathbb{R}$ the corresponding shear velocity at $t=0$ and by $s \zeta(t)$, with $\zeta(0)=1$, its velocity at any instant $t \in[0, \tau], \tau>0$. We introduce a function $g_{0}: \partial \Omega \rightarrow \mathbb{R}^{2}$ such that

$$
\begin{aligned}
& \int_{\Gamma_{L}} g_{0} \cdot n d \sigma=0, \quad g_{0}=0 \text { on } \Gamma_{1}, \\
& g_{0 n}=g_{0} \cdot n=0 \text { and } g_{0 \mathcal{T}}=g_{0}-g_{0 n} n=(s, 0) \text { on } \Gamma_{0},
\end{aligned}
$$

where $n=\left(n_{1}, n_{2}\right)$ is the unit outward normal vector to $\partial \Omega$ and $g_{0} \cdot n$ is the Euclidean inner product of the vectors $g_{0}$ and $n$.

Then the fluid flow satisfies the following non-homogeneous boundary conditions on $\Gamma_{1} \cup \Gamma_{L}$

$$
v=0 \text { on } \Gamma_{1} \times(0, \tau), \quad v=g_{0} \zeta \text { on } \Gamma_{L} \times(0, \tau),
$$

and a slip condition on $\Gamma_{0}$

$$
v_{n}=v \cdot n=0 \text { on } \Gamma_{0} \times(0, \tau),
$$


combined with Tresca's friction law for the unknown tangential velocity $\left({ }^{28}\right)$

$$
\begin{aligned}
& \left|\sigma_{\mathcal{T}}\right|<\ell \Rightarrow v_{\mathcal{T}}=v-v_{n} n=(s \zeta, 0) \\
& \left|\sigma_{\mathcal{T}}\right|=\ell \Rightarrow \exists \lambda \geq 0 \text { such that } v_{\mathcal{T}}=(s \zeta, 0)-\lambda \sigma_{\mathcal{T}}
\end{aligned}
$$

where $\ell$ is the upper limit for the shear stress (i.e. $\ell$ is the Tresca's friction threshold) and

$$
\sigma_{\mathcal{T}}=\left(\sigma_{i j} n_{j}-\sigma_{n} n_{i}\right)_{1 \leq i \leq 2}, \sigma_{n}=\sigma_{i j} n_{i} n_{j}
$$

Note that we will use Einstein's summation convention throughout the paper.

Finally we prescribe also non-homogeneous boundary conditions for the temperature i.e.

$$
T=g \text { on } \partial \Omega \times(0, \tau) .
$$

Let us emphasize that whenever the thermal capacity and the dissipative term $\phi$ do not depend on the velocity field, the heat equation can be solved independently of the fluid flow problem and the temperature-dependent viscosity can be considered as a data. In this simplified case, an existence result has been proved in ${ }^{29}$ and uniqueness and regularity properties have been obtained in ${ }^{30}$. Hence some fixed point technique appears as the most natural tool to study our coupled problem $\left({ }^{31}\right)$. Nevertheless, motivated by computational issues, we will follow in this paper another strategy. Indeed, observing that for any given time-independent viscosity and Tresca's threshold, the Navier-Stokes system can be solved numerically with standard softwares, we propose to split the whole time-interval $[0, \tau]$ into a finite family of subintervals, i.e. $[0, \tau]=\cup_{n=0}^{N-1}\left[t_{n}, t_{n+1}\right]$, and to replace on each subinterval $\left[t_{n}, t_{n+1}\right]$ the viscosity $\mu(T)$ and the Tresca's threshold $\ell(x, t)$ by some appropriate time-independent approximations $\mu_{n}(x)$, $\ell_{n}(x)$. Similarly, we will replace the thermal capacity and conductivity, $c(v)$ and $K(x, t)$, by some approximations $c_{n}(x)$ and $K_{n}(x)$, in order to get a simplified heat problem on $\left[t_{n}, t_{n+1}\right]$. By using this time-splitting method, we replace the coupled problem $(P)$ by a finite family of decoupled fluid flow/heat transfer problems which are easy to solve numerically and we will show the convergence of the corresponding approximate solutions to a solution of $(P)$ when the length of the subintervals tends to zero.

The paper is organized as follows. In Section 2 we give the mathematical formulation of the coupled problem $(P)$. Then in Section 3, we introduce the time-splitting technique and we show that the decoupled fluid flow/heat transfer problems admit time-continuous solutions. This property will allow us to define a sequence of approximate solutions $\left(v_{h}, p_{h}, T_{h}\right)$ on the whole time-interval $[0, \tau]$, where $h$ denotes the length of each subinterval. In Section 4, we establish some a priori estimates for $\left(v_{h}, p_{h}, T_{h}\right)$ and we prove the convergence to a solution of $(P)$. Finally, in Section 5 we illustrate these theoretical results with an example of implementation.

\section{Formulation of the coupled fluid flow / heat transfer problem}

We adopt the same notations as in $^{29}$ for the formulation of the fluid flow problem. More precisely, we denote by

$$
\begin{aligned}
& \mathbf{L}^{2}(\Omega)=\left(L^{2}(\Omega)\right)^{2}, \mathbf{L}^{4}(\Omega)=\left(L^{4}(\Omega)\right)^{2}, \mathbf{L}^{2}\left(\Gamma_{0}\right)=\left(L^{2}\left(\Gamma_{0}\right)\right)^{2} \\
& \mathbf{H}^{1}(\Omega)=\left(H^{1}(\Omega)\right)^{2}, \mathbf{H}^{2}(\Omega)=\left(H^{2}(\Omega)\right)^{2}
\end{aligned}
$$


and we define

$$
\mathcal{V}_{0}=\left\{\varphi \in \mathbf{H}^{1}(\Omega): \varphi=0 \text { on } \Gamma_{1} \cup \Gamma_{L}, \varphi \cdot n=0 \text { on } \Gamma_{0}\right\}
$$

endowed with the norm of $\mathbf{H}^{1}(\Omega)$ and

$$
\mathcal{V}_{0 d i v}=\left\{\varphi \in \mathcal{V}_{0}: \operatorname{div}(\varphi)=0\right\}
$$

We assume that

$$
\begin{aligned}
& f \in L^{2}\left(0, \tau ; \mathbf{L}^{2}(\Omega)\right), \\
& \ell \in W^{1,2}\left(0, \tau ; L_{+}^{2}\left(\Gamma_{0}\right)\right) \cap C^{0}\left([0, \tau] ; L_{+}^{\infty}\left(\Gamma_{0}\right)\right), \\
& \zeta \in \mathcal{C}^{\infty}([0, \tau]) \text { with } \zeta(0)=1
\end{aligned}
$$

and $\tau>0$. We assume moreover that $\mu \in \mathcal{C}^{1}(\mathbb{R}, \mathbb{R})$ and there exist three real numbers $\mu^{*}, \mu_{*}$ and $\mu_{*}^{\prime}$ such that

$$
0<\mu^{*} \leq 2 \mu(X) \leq \mu_{*}, \quad\left|\mu^{\prime}(X)\right| \leq \mu_{*}^{\prime} \quad \forall X \in \mathbb{R} .
$$

Then, for any measurable temperature field $T$ we define

$$
\begin{aligned}
a(T ; \cdot, \cdot) \quad: \quad L^{2}\left(0, \tau ; \mathbf{H}^{1}(\Omega)\right) \times L^{2}\left(0, \tau ; \mathbf{H}^{1}(\Omega)\right) \rightarrow \mathbb{R} \\
(u, v) \mapsto \int_{0}^{\tau} \int_{\Omega} 2 \mu(T) D_{i j}(u) D_{i j}(v) d x d t .
\end{aligned}
$$

Let $b$ be the usual trilinear form and $\Psi$ be the Tresca's functional given by

$$
\begin{aligned}
b: & \mathbf{H}^{1}(\Omega) \times \mathbf{H}^{1}(\Omega) \times \mathbf{H}^{1}(\Omega) \rightarrow \mathbb{R} \\
(u, v, w) & \mapsto \int_{\Omega} u_{i} \frac{\partial v_{j}}{\partial x_{i}} w_{j} d x
\end{aligned}
$$

and

$$
\begin{aligned}
\Psi \quad: \quad L^{2}\left(0, \tau ; \mathbf{L}^{2}\left(\Gamma_{0}\right)\right) \rightarrow \mathbb{R} \\
u \mapsto \int_{0}^{\tau} \int_{\Gamma_{0}} \ell|u| d x_{1} d t .
\end{aligned}
$$

For the heat problem, we assume that the thermal conductivity $K$ is a symmetric matrix such that

$$
K_{i j} \in W^{1, \infty}\left(0, \tau ; W^{1, \infty}(\Omega)\right) \quad \forall i, j \in\{1,2\}
$$

and there exists $\alpha^{*}>0$ such that

$$
K_{i j}(x, t) \xi_{i} \xi_{j} \geq \alpha^{*}\left|\xi_{i}\right|^{2} \forall \text { a.e }(x, t) \in \Omega \times(0, \tau), \forall \xi \in \mathbb{R}^{2} .
$$

We assume also that $d$ is a positive constant, $c$ and $\phi$ are two Lipschitz continuous mappings and there exist $c_{*}, c^{*}$ two real numbers such that

$$
0<c^{*} \leq c(X) \leq c_{*} \quad \forall X \in \mathbb{R}^{2} .
$$


Finally, we assume that there exist an extension of $g_{0}$ and $g$ to $\Omega$, denoted respectively by $G_{0}$ and $G$, such that

$$
\begin{aligned}
& G_{0} \in \mathbf{H}^{2}(\Omega), \operatorname{div}\left(G_{0}\right)=0 \text { in } \Omega, G_{0}=g_{0} \text { on } \Gamma_{L}, \\
& G_{0}=0 \text { on } \Gamma_{1}, G_{0 n}=0 \text { and } G_{0 \mathcal{T}}=(s, 0) \text { on } \Gamma_{0}
\end{aligned}
$$

and

$$
G \in C^{2}\left([0, \tau] ; H^{2}(\Omega)\right), G=g \text { on } \partial \Omega \times[0, \tau] .
$$

In order to deal with Dirichlet homogeneous boundary conditions, we set $\widetilde{v}=v-G_{0} \zeta$ and $\widetilde{T}=$ $T-G$. The variational formulation of the coupled problem is given by

Problem (P) Find a triplet velocity-pressure-temperature $(\widetilde{v}, p, \widetilde{T})$ with

$$
\widetilde{v} \in L^{2}\left(0, \tau ; \mathcal{V}_{0 d i v}\right) \cap L^{\infty}\left(0, \tau ; \mathbf{L}^{2}(\Omega)\right), p \in H^{-1}\left(0, \tau ; L_{0}^{2}(\Omega)\right)
$$

and

$$
\widetilde{T} \in L^{2}\left(0, \tau ; H_{0}^{1}(\Omega)\right), \frac{\partial \widetilde{T}}{\partial t} \in L^{2}\left(0, \tau ; L^{2}(\Omega)\right), \frac{\partial^{2} \widetilde{T}}{\partial t^{2}} \in L^{2}\left(0, \tau ; H^{-1}(\Omega)\right)
$$

such that

$$
\begin{aligned}
& \text { Problem }\left(\mathbf{P}_{\text {flow }}\right): \text { for all } \varphi \in \mathcal{V}_{0}, \text { for all } \chi \in \mathcal{D}(0, \tau) \\
& \left\langle\frac{d}{d t}(\widetilde{v}, \varphi)+b(\widetilde{v}, \widetilde{v}, \varphi), \chi\right\rangle_{\mathcal{D}^{\prime}(0, \tau), \mathcal{D}(0, \tau)}+a(\widetilde{T}+G ; \widetilde{v}, \varphi \chi) \\
& -\left\langle\int_{\Omega} p \operatorname{div}(\varphi) d x, \chi\right\rangle_{\mathcal{D}^{\prime}(0, \tau), \mathcal{D}(0, \tau)}+\Psi(\widetilde{v}+\varphi \chi)-\Psi(\widetilde{v}) \\
& \geq\left\langle\left(f-G_{0} \frac{\partial \zeta}{\partial t}, \varphi\right), \chi\right\rangle_{\mathcal{D}^{\prime}(0, \tau), \mathcal{D}(0, \tau)}-a\left(\widetilde{T}+G ; G_{0} \zeta, \varphi \chi\right) \\
& -\left\langle b\left(G_{0} \zeta, \widetilde{v}+G_{0} \zeta, \varphi\right)+b\left(\widetilde{v}, G_{0} \zeta, \varphi\right), \chi\right\rangle_{\mathcal{D}^{\prime}(0, \tau), \mathcal{D}(0, \tau)}
\end{aligned}
$$

where $(.,$.$) denotes the inner product in \mathbf{L}^{2}(\Omega)$, coupled with

Problem $\left(\mathbf{P}_{\text {heat }}\right)$ : for all $w \in L^{2}\left(0, \tau ; H_{0}^{1}(\Omega)\right)$

$$
\begin{aligned}
& d \int_{0}^{\tau}\left\langle\frac{\partial^{2} \widetilde{T}}{\partial t^{2}}, w\right\rangle_{H^{-1}(\Omega), H_{0}^{1}(\Omega)} d t+\int_{0}^{\tau} \int_{\Omega} c\left(\widetilde{v}+G_{0} \zeta\right) \frac{\partial \widetilde{T}}{\partial t} w d x d t+\int_{0}^{\tau}(K \nabla \widetilde{T}, \nabla w) d t \\
& =\int_{0}^{\tau} \int_{\Omega}^{\tau} \phi\left(\widetilde{v}+G_{0} \zeta\right) w d x d t-d \int_{0}^{\tau} \int_{\Omega} \frac{\partial^{2} G}{\partial t^{2}} w d x d t-\int_{0}^{\tau} \int_{\Omega} c\left(\widetilde{v}+G_{0} \zeta\right) \frac{\partial G}{\partial t} w d x d t \\
& -\int_{0}^{\tau}(K \nabla G, \nabla w) d t
\end{aligned}
$$

and satisfying the initial conditions

$$
\begin{aligned}
& \widetilde{v}(\cdot, 0)=\widetilde{v}_{0}=v_{0}-G_{0} \in \mathbf{L}^{2}(\Omega), \\
& \widetilde{T}(\cdot, 0)=\widetilde{T}_{0}=T_{0}-G(\cdot, 0) \in H_{0}^{1}(\Omega), \frac{\partial \widetilde{T}}{\partial t}(\cdot, 0)=\widetilde{T}_{1}=T_{1}-\frac{\partial G}{\partial t}(\cdot, 0) \in L^{2}(\Omega) .
\end{aligned}
$$




\section{Description of the time-splitting technique}

Now let us describe our time-splitting technique. Let $N \in \mathbb{N}^{*}$. We decompose the time interval $[0, \tau]$ into $N$ subintervals $\left[t_{n}, t_{n+1}\right]$ of length $h=\frac{\tau}{N}$ with $t_{k}=k h$ for all $k \in\{0, \ldots, N\}$. On each subinterval $\left[t_{n}, t_{n+1}\right], 0 \leq n \leq N-1$, we consider the following approximate problems

Problem $\left(\mathbf{P}_{\mathbf{h} \text { flow }}\right)$ Find

$$
\widetilde{v}_{h}^{n} \in L^{2}\left(t_{n}, t_{n+1} ; \mathcal{V}_{0 d i v}\right) \cap L^{\infty}\left(t_{n}, t_{n+1} ; \mathbf{L}^{2}(\Omega)\right), p_{h}^{n} \in H^{-1}\left(t_{n}, t_{n+1} ; L_{0}^{2}(\Omega)\right)
$$

such that, for all $\varphi \in \mathcal{V}_{0}$ and $\chi \in \mathcal{D}\left(t_{n}, t_{n+1}\right)$, we have

$$
\begin{aligned}
& \left\langle\frac{d}{d t}\left(\widetilde{v}_{h}^{n}, \varphi\right)+b\left(\widetilde{v}_{h}^{n}, \widetilde{v}_{h}^{n}, \varphi\right), \chi\right\rangle_{\mathcal{D}^{\prime}\left(t_{n}, t_{n+1}\right), \mathcal{D}\left(t_{n}, t_{n+1}\right)}+a_{n}\left(\widetilde{v}_{h}^{n}, \varphi \chi\right) \\
& -\left\langle\int_{\Omega} p_{h}^{n} \operatorname{div}(\varphi) d x, \chi\right\rangle_{\mathcal{D}^{\prime}\left(t_{n}, t_{n+1}\right), \mathcal{D}\left(t_{n}, t_{n+1}\right)}+\Psi_{n}\left(\widetilde{v}_{h}^{n}+\varphi \chi\right)-\Psi_{n}\left(\widetilde{v}_{h}^{n}\right) \\
& \geq\left\langle\left(f-G_{0} \frac{\partial \zeta}{\partial t}, \varphi\right), \chi\right\rangle_{\mathcal{D}^{\prime}\left(t_{n}, t_{n+1}\right), \mathcal{D}\left(t_{n}, t_{n+1}\right)}-a_{n}\left(G_{0} \zeta, \varphi \chi\right) \\
& -\left\langle b\left(G_{0} \zeta, \widetilde{v}_{h}^{n}+G_{0} \zeta, \varphi\right)+b\left(\widetilde{v}_{h}^{n}, G_{0} \zeta, \varphi\right), \chi\right\rangle_{\mathcal{D}^{\prime}\left(t_{n}, t_{n+1}\right), \mathcal{D}\left(t_{n}, t_{n+1}\right)}
\end{aligned}
$$

with the initial condition

$$
\widetilde{v}_{h}^{n}\left(\cdot, t_{n}\right)=\widetilde{v}_{h, 0}^{n} \in \mathbf{L}^{2}(\Omega)
$$

and

Problem $\left(\mathbf{P}_{\mathbf{h} \text { heat }}^{\mathbf{n}}\right)$ Find

$$
\widetilde{T}_{h}^{n} \in L^{2}\left(t_{n}, t_{n+1} ; H_{0}^{1}(\Omega)\right), \frac{\partial \widetilde{T}_{h}^{n}}{\partial t} \in L^{2}\left(t_{n}, t_{n+1} ; L^{2}(\Omega)\right), \frac{\partial^{2} \widetilde{T}_{h}^{n}}{\partial t^{2}} \in L^{2}\left(t_{n}, t_{n+1} ; H^{-1}(\Omega)\right)
$$

such that, for all for all $w \in L^{2}\left(t_{n}, t_{n+1} ; H_{0}^{1}(\Omega)\right)$, we have

$$
\begin{aligned}
& d \int_{t_{n}}^{t_{n+1}}\left\langle\frac{\partial^{2} \widetilde{T}_{h}^{n}}{\partial t^{2}}, w\right\rangle_{H^{-1}(\Omega), H_{0}^{1}(\Omega)}^{t_{n+1}} d t+\int_{t_{n}}^{t_{n+1}} \int_{\Omega} c_{n} \frac{\partial \widetilde{T}_{h}^{n}}{\partial t} w d x d t+\int_{t_{n}}^{t_{n+1}}\left(K_{n} \nabla \widetilde{T}_{h}^{n}, \nabla w\right) d t \\
& =\int_{t_{n}}^{t_{n+1}} \int_{\Omega} \phi_{n} w d x d t-d \int_{t_{n}}^{t^{2}} \int_{\Omega} \frac{\partial^{2}}{\partial t^{2}} d x-\int_{t_{n}}^{t_{n+1}} \int_{\Omega} c_{n} \frac{\partial G}{\partial t} w d x d t \\
& -\int_{t_{n}}^{t_{n+1}}\left(K_{n} \nabla G, \nabla w\right) d t
\end{aligned}
$$

with the initial conditions

$$
\widetilde{T}_{h}^{n}\left(\cdot, t_{n}\right)=\widetilde{T}_{h, 0}^{n} \in H_{0}^{1}(\Omega), \frac{\partial \widetilde{T}_{h}^{n}}{\partial t}\left(\cdot, t_{n}\right)=\widetilde{T}_{h, 1}^{n} \in L^{2}(\Omega),
$$


where $c_{n} \in L^{\infty}\left(t_{n}, t_{n+1} ; L^{\infty}(\Omega)\right), \phi_{n} \in L^{2}\left(t_{n}, t_{n+1} ; L^{2}(\Omega)\right), \widetilde{v}_{h, 0}^{n}, \widetilde{T}_{h, 0}^{n}, \widetilde{T}_{h, 1}^{n}$ will be defined later on and

$$
\begin{gathered}
a_{n}: \quad L^{2}\left(t_{n}, t_{n+1} ; \mathbf{H}^{1}(\Omega)\right) \times L^{2}\left(t_{n}, t_{n+1} ; \mathbf{H}^{1}(\Omega)\right) \rightarrow \mathbb{R} \\
(u, v) \mapsto \int_{t_{n}}^{t_{n+1}} \int_{\Omega} 2 \mu_{n} D_{i j}(u) D_{i j}(v) d x d t
\end{gathered}
$$

with $\mu_{n}=\mu\left(\widetilde{T}_{h, 0}^{n}+G\left(\cdot, t_{n}\right)\right)$,

$$
\begin{aligned}
\Psi_{n}: & L^{2}\left(t_{n}, t_{n+1} ; \mathbf{L}^{2}\left(\Gamma_{0}\right)\right) \rightarrow \mathbb{R} \\
& u \mapsto \int_{t_{n}}^{t_{n+1}} \int_{\Gamma_{0}} \ell_{n}|u| d x_{1} d t
\end{aligned}
$$

with $\ell_{n}=\ell\left(\cdot, t_{n}\right)$ and $K_{n}=K\left(\cdot, t_{n}\right)$. Let us emphazise that the problems $\left(\mathrm{P}_{\mathrm{h} \mathrm{flow}}^{\mathrm{n}}\right)-\left(\mathrm{P}_{\mathrm{h} \text { heat }}^{\mathrm{n}}\right)$ are totally decoupled. Furthermore, by using Theorem 6.2 in $^{29}$, Lemma 3.1 and Proposition 3.2 in $^{30}$ we have

Theorem 3.1. (Existence and uniqueness result for $\left(\mathrm{P}_{\mathrm{h} \text { flow }}^{\mathrm{n}}\right)$ ). Assume that (6)-(7) and (11) hold. Let $N \in \mathbb{N}^{*}$ and $n \in\{0, \ldots, N-1\}$. Then, for any $\widetilde{v}_{h, 0}^{n} \in \mathbf{L}^{2}(\Omega)$ and $\widetilde{T}_{h, 0}^{n} \in H_{0}^{1}(\Omega)$, problem $\left(\mathrm{P}_{\mathrm{h} \text { flow }}^{\mathrm{n}}\right)$ admits an unique solution. Furthermore $\frac{\partial \widetilde{v}_{h}^{n}}{\partial t}$ and $b\left(\widetilde{v}_{h}^{n}, \widetilde{v}_{h}^{n}, \cdot\right)$ belong to $L^{2}\left(t_{n}, t_{n+1},\left(\mathcal{V}_{0 d i v}\right)^{\prime}\right)$.

It follows that $\widetilde{v}_{h}^{n} \in C^{0}\left(\left[t_{n}, t_{n+1}\right] ; \mathbf{L}^{2}(\Omega)\right)$ and for all $\varphi \in \mathcal{V}_{0 \text { div }}$ and $\chi \in C^{\infty}\left(\left[t_{n}, t_{n+1}\right]\right)$, we have

$$
\begin{aligned}
& \int_{t_{n}}^{t_{n+1}}\left\langle\frac{\partial \widetilde{v}_{h}^{n}}{\partial t}, \varphi\right\rangle_{\left(\mathcal{V}_{0 d i v}\right)^{\prime}, \mathcal{V}_{0 d i v}} \chi d t+\int_{t_{n}}^{t_{n+1}} b\left(\widetilde{v}_{h}^{n}, \widetilde{v}_{h}^{n}, \varphi\right) \chi d t+a_{n}\left(\widetilde{v}_{h}^{n}, \varphi \chi\right) \\
& +\Psi_{n}\left(\widetilde{v}_{h}^{n}+\varphi \chi\right)-\Psi_{n}\left(\widetilde{v}_{h}^{n}\right) \geq \int_{t_{n}}^{t_{n+1}}\left(f-G_{0} \frac{\partial \zeta}{\partial t}, \varphi\right) \chi d t-a_{n}\left(G_{0} \zeta, \varphi \chi\right) \\
& -\int_{t_{n}}^{t_{n+1}}\left(b\left(G_{0} \zeta, \widetilde{v}_{h}^{n}+G_{0} \zeta, \varphi\right)+b\left(\widetilde{v}_{h}^{n}, G_{0} \zeta, \varphi\right)\right) \chi d t .
\end{aligned}
$$

We may also obtain an existence result for $\left(\mathrm{P}_{\mathrm{h} \text { heat }}^{\mathrm{n}}\right)$.

Proposition 3.2. (Existence and uniqueness result for $\left(\mathrm{P}_{\mathrm{h} \text { heat }}^{\mathrm{n}}\right)$ ). Assume that (8)-(9) and (12) hold. Let $N \geq\left\lfloor\frac{c_{*}^{2} \tau \exp (\tau)}{d^{2}}\right\rfloor+1, n \in\{0, \ldots, N-1\}$ and let us assume moreover that

$$
c_{n} \in L^{\infty}\left(t_{n}, t_{n+1} ; L^{\infty}(\Omega)\right), \phi_{n} \in L^{2}\left(t_{n}, t_{n+1} ; L^{2}(\Omega)\right)
$$

with

$$
0<c^{*} \leq c_{n}(x, t) \leq c_{*} \forall \text { a.e }(x, t) \in \Omega \times\left(t_{n}, t_{n+1}\right)
$$

and

$$
\widetilde{T}_{h, 0}^{n} \in H_{0}^{1}(\Omega), \widetilde{T}_{h, 1}^{n} \in L^{2}(\Omega) .
$$

Then problem $\left(\mathrm{P}_{\mathrm{h} \text { heat }}^{\mathrm{n}}\right)$ admits an unique solution $\widetilde{T}_{h}^{n}$ such that $\widetilde{T}_{h}^{n} \in C^{0}\left(\left[t_{n}, t_{n+1}\right] ; H_{0}^{1}(\Omega)\right) \cap$ $C^{1}\left(\left[t_{n}, t_{n+1}\right] ; L^{2}(\Omega)\right)$. 
Remark 3.3. The condition $N \geq\left\lfloor\frac{c_{*}^{2} \tau \exp (\tau)}{d^{2}}\right\rfloor+1$, ensures that the length $h=\frac{\tau}{N}$ of the subintervals $\left[t_{n}, t_{n+1}\right], n \in\{0, \ldots, N-1\}$, satisfies the inequality

$$
\exp (h) h<\left(\frac{d}{c_{*}}\right)^{2}
$$

where $d$ is the damping coefficient and $c_{*}$ the maximal value of the thermal capacity (see (10)). Hence the limitation on the length of the small time-intervals depends only on the data.

Proof. Let $N \in \mathbb{N}^{*}$ and $n \in\{0, \ldots, N-1\}$. We introduce the functional space

$$
W=C^{0}\left(\left[t_{n}, t_{n+1}\right] ; H_{0}^{1}(\Omega)\right) \cap C^{1}\left(\left[t_{n}, t_{n+1}\right] ; L^{2}(\Omega)\right)
$$

endowed with the norm

$$
\|\varphi\|_{W}=\sup _{t \in\left[t_{n}, t_{n+1}\right]}\left(d\left\|\frac{\partial \varphi}{\partial t}(\cdot, t)\right\|_{L^{2}(\Omega)}^{2}+\left(K_{n} \nabla \varphi(\cdot, t), \nabla \varphi(\cdot, t)\right)\right)^{\frac{1}{2}}
$$

for all $\varphi \in W$. With theorems 8.1 and 8.2, in ${ }^{32}$ chapter 3, we know that, for any $u_{0}^{n} \in H_{0}^{1}(\Omega)$, $u_{1}^{n} \in L^{2}(\Omega)$ and $F \in L^{2}\left(t_{n}, t_{n+1} ; L^{2}(\Omega)\right)$, the problem

$$
\left\{\begin{array}{l}
d \frac{\partial^{2} u}{\partial t^{2}}-\operatorname{div}\left(K_{n} \nabla u\right)=F \text { in } \Omega \\
u=0 \text { on } \partial \Omega \\
u\left(\cdot, t_{n}\right)=u_{0}^{n}, \frac{\partial u}{\partial t}\left(\cdot, t_{n}\right)=u_{1}^{n}
\end{array}\right.
$$

admits an unique solution $u \in W$. Furthermore (see Lemma 8.3, in ${ }^{32}$ chapter 3),

$$
\begin{aligned}
& d\left\|\frac{\partial u}{\partial t}(\cdot, s)\right\|_{L^{2}(\Omega)}^{2}+\left(K_{n} \nabla u(\cdot, s), \nabla u(\cdot, s)\right)=d\left\|u_{1}^{n}\right\|_{L^{2}(\Omega)}^{2} \\
& +\left(K_{n} \nabla u_{0}^{n}, \nabla u_{0}^{n}\right)+2 \int_{t_{n}}^{s} \int_{\Omega} F \frac{\partial u}{\partial t} d x d t
\end{aligned}
$$

for all $s \in\left[t_{n}, t_{n+1}\right]$.

So we define the fixed point mapping

$$
\Sigma:\left\{\begin{array}{l}
W \rightarrow W \\
\widetilde{u} \longmapsto u
\end{array}\right.
$$

where $u$ is solution of (13) with $u_{0}^{n}=\widetilde{T}_{h, 0}^{n}, u_{1}^{n}=\widetilde{T}_{h, 1}^{n}$ and

$$
F=\phi_{n}-d \frac{\partial^{2} G}{\partial t^{2}}-c_{n} \frac{\partial G}{\partial t}+\operatorname{div}\left(K_{n} \nabla G\right)-c_{n} \frac{\partial \widetilde{u}}{\partial t} .
$$


The mapping $\Sigma$ is Lipschitz continuous on $W$. Indeed, for any $\widetilde{u}_{1}$ and $\widetilde{u}_{2}$ in $W$, let $u=\Sigma\left(\widetilde{u}_{1}\right)-\Sigma\left(\widetilde{u}_{2}\right)$. Then $u$ is solution of (13) with $u_{0}^{n}=u_{1}^{n}=0$ and $F=c_{n} \frac{\partial\left(\widetilde{u}_{2}-\widetilde{u}_{1}\right)}{\partial t}$. With (14) we infer that

$$
\begin{aligned}
& d\left\|\frac{\partial u}{\partial t}(\cdot, s)\right\|_{L^{2}(\Omega)}^{2}+\left(K_{n} \nabla u(\cdot, s), \nabla u(\cdot, s)\right) \\
& \leq \frac{c_{*}^{2}}{d} \int_{t_{n}}^{s}\left\|\frac{\partial\left(\widetilde{u}_{1}-\widetilde{u}_{2}\right)}{\partial t}(\cdot, t)\right\|_{L^{2}(\Omega)}^{2} d t+d \int_{t_{n}}^{s}\left\|\frac{\partial u}{\partial t}(\cdot, t)\right\|_{L^{2}(\Omega)}^{2} d t
\end{aligned}
$$

for all $s \in\left[t_{n}, t_{n+1}\right]$. By applying Grönwall's lemma, we get

$$
\|u\|_{W}^{2} \leq \frac{c_{*}^{2} \exp (h)}{d} \int_{t_{n}}^{t_{n+1}}\left\|\frac{\partial\left(\widetilde{u}_{1}-\widetilde{u}_{2}\right)}{\partial t}(\cdot, t)\right\|_{L^{2}(\Omega)}^{2} d t \leq \frac{c_{*}^{2} \exp (h) h}{d^{2}}\left\|\widetilde{u}_{1}-\widetilde{u}_{2}\right\|_{W}^{2} .
$$

It follows $\Sigma$ is a contraction whenever $N \geq\left\lfloor\frac{c_{*}^{2} \tau \exp (\tau)}{d^{2}}\right\rfloor+1$, thus $\Sigma$ admits an unique fixed point in $W$ which allows us to conclude.

Let us assume from now on that

$$
N \geq\left\lfloor\frac{c_{*}^{2} \tau \exp (\tau)}{d^{2}}\right\rfloor+1
$$

Our time-splitting technique is based on the so-called time retardation method, namely we split the whole time interval $[0, \tau]$ into the small intervals $\left[t_{n}, t_{n+1}\right]$ with $h=\frac{\tau}{N}$ and $t_{n}=n h$ for all $n \in\{0, \ldots, N\}$ and we solve on each subinterval $\left[t_{n}, t_{n+1}\right]$ the approximate problems $\left(\mathrm{P}_{\mathrm{h} \mathrm{flow}}^{\mathrm{n}}\right)-\left(\mathrm{P}_{\mathrm{h} \text { heat }}^{\mathrm{n}}\right)$ where the value of the unknows $v$ and $T$ from the previous subinterval is considered in the coupling terms.

More precisely we can choose

$$
\begin{aligned}
& \widetilde{v}_{h, 0}^{n}=\widetilde{v}_{h}^{n-1}\left(\cdot, t_{n}\right), \\
& \widetilde{T}_{h, 0}^{n}=\widetilde{T}_{h}^{n-1}\left(\cdot, t_{n}\right), \widetilde{T}_{h, 1}^{n}=\frac{\partial \widetilde{T}_{h}^{n-1}}{\partial t}\left(\cdot, t_{n}\right)
\end{aligned}
$$

for all $n \in\{1, \ldots, N-1\}$ and we let

$$
\begin{aligned}
& \widetilde{v}_{h, 0}^{0}=\widetilde{v}_{0} \in \mathbf{L}^{2}(\Omega), \\
& \widetilde{T}_{h, 0}^{0}=\widetilde{T}_{0} \in H_{0}^{1}(\Omega), \widetilde{T}_{h, 1}^{0}=\widetilde{T}_{1} \in L^{2}(\Omega) .
\end{aligned}
$$

In order to complete the description of the approximate problems $\left(\mathrm{P}_{\mathrm{h} \text { flow }}^{\mathrm{n}}\right)-\left(\mathrm{P}_{\mathrm{h} \text { heat }}^{\mathrm{n}}\right)$, we define the mappings $c_{n}$ and $\phi_{n}$ as follows

$$
c_{0}=c\left(\widetilde{v}_{0}+G_{0}\right), \phi_{0}=\phi\left(\widetilde{v}_{0}+G_{0}\right)
$$

and, for all $n \in\{1, \ldots, N-1\}$

$c_{n}(\cdot, t)=c\left(\widetilde{v}_{h}^{n-1}(\cdot, t-h)+G_{0}(\cdot) \zeta(t)\right), \quad \phi_{n}(\cdot, t)=\phi\left(\widetilde{v}_{h}^{n-1}(\cdot, t-h)+G_{0}(\cdot) \zeta(t)\right) \quad \forall t \in\left[t_{n}, t_{n+1}\right]$. 
Then, under the assumptions (6)-(12), (15) and (16), we can define by induction $\widetilde{v}_{h}^{n}, p_{h}^{n}$ and $\widetilde{T}_{h}^{n}$ for all $n \in\{0, \ldots, N-1\}$ and we have

$$
\begin{aligned}
& \widetilde{v}_{h}^{n} \in L^{2}\left(t_{n}, t_{n+1} ; \mathcal{V}_{0 d i v}\right) \cap C^{0}\left(\left[t_{n}, t_{n+1}\right] ; \mathbf{L}^{2}(\Omega)\right), \frac{\partial \widetilde{v}_{h}^{n}}{\partial t} \in L^{2}\left(t_{n}, t_{n+1} ;\left(\mathcal{V}_{0 \text { div }}\right)^{\prime}\right), \\
& \widetilde{T}_{h}^{n} \in C^{0}\left(\left[t_{n}, t_{n+1}\right] ; H_{0}^{1}(\Omega)\right) \cap C^{1}\left(\left[t_{n}, t_{n+1}\right] ; L^{2}(\Omega)\right), \frac{\partial^{2} \widetilde{T}_{h}^{n}}{\partial t^{2}} \in L^{2}\left(t_{n}, t_{n+1} ; H^{-1}(\Omega)\right) .
\end{aligned}
$$

Then we let

$$
\widetilde{v}_{h}(\cdot, t)=\widetilde{v}_{h}^{n}(\cdot, t), \widetilde{T}_{h}(\cdot, t)=\widetilde{T}_{h}^{n}(\cdot, t) \quad \forall t \in\left[t_{n}, t_{n+1}\right],
$$

for all $n \in\{0, \ldots, N-1\}$. We have

$$
\begin{aligned}
& \widetilde{v}_{h} \in L^{2}\left(0, \tau ; \mathcal{V}_{0 \text { div }}\right) \cap C^{0}\left([0, \tau] ; \mathbf{L}^{2}(\Omega)\right), \frac{\partial \widetilde{v}_{h}}{\partial t} \in L^{2}\left(0, \tau ;\left(\mathcal{V}_{0 \text { div }}\right)^{\prime}\right), \\
& \widetilde{T}_{h} \in C^{0}\left([0, \tau] ; H_{0}^{1}(\Omega)\right) \cap C^{1}\left([0, \tau] ; L^{2}(\Omega)\right), \frac{\partial^{2} \widetilde{T}_{h}}{\partial t^{2}} \in L^{2}\left(0, \tau ; H^{-1}(\Omega)\right)
\end{aligned}
$$

such that

$$
\begin{aligned}
& \int_{0}^{\tau}\left\langle\frac{\partial \widetilde{v}_{h}}{\partial t}, \varphi\right\rangle_{\left(\mathcal{V}_{0 d i v}\right)^{\prime}, \mathcal{V}_{0 d i v}} \chi d t+\int_{0}^{\tau} b\left(\widetilde{v}_{h}, \widetilde{v}_{h}, \varphi\right)+a_{h}\left(\widetilde{v}_{h}, \varphi \chi\right) \chi d t \\
& +\Psi_{h}\left(\widetilde{v}_{h}+\varphi \chi\right)-\Psi_{h}\left(\widetilde{v}_{h}\right) \geq \int_{0}^{\tau}\left(f-G_{0} \frac{\partial \zeta}{\partial t}, \varphi\right) \chi d t-a_{h}\left(G_{0} \zeta, \varphi \chi\right) \\
& -\int_{0}^{\tau}\left(b\left(G_{0} \zeta, \widetilde{v}_{h}+G_{0} \zeta, \varphi\right)+b\left(\widetilde{v}_{h}, G_{0} \zeta, \varphi\right)\right) \chi d t \quad \forall \varphi \in \mathcal{V}_{0 d i v}, \forall \chi \in \mathcal{D}(0, \tau)
\end{aligned}
$$

and

$$
\begin{aligned}
& d \int_{0}^{\tau}\left\langle\frac{\partial^{2} \widetilde{T}_{h}}{\partial t^{2}}, w\right\rangle \quad d t+\int_{0}^{\tau} \int_{\Omega} c_{h} \frac{\partial \widetilde{T}_{h}}{\partial t} w d x d t+\int_{0}^{\tau}\left(K_{h} \nabla \widetilde{T}_{h}, \nabla w\right) d t \\
& =\int_{0}^{\tau} \int_{\Omega} \phi_{h} w d x d t-d \int_{0}^{\tau} \int_{\Omega}^{\tau} \frac{\partial^{1} G}{\partial t^{2}} w d x d t-\int_{0}^{\tau} \int_{\Omega} c_{h} \frac{\partial G}{\partial t} w d x d t \\
& -\int_{0}^{\tau}\left(K_{h} \nabla G, \nabla w\right) d t \quad \forall w \in L^{2}\left(0, \tau ; H_{0}^{1}(\Omega)\right)
\end{aligned}
$$

with the initial conditions

$$
\begin{aligned}
& \widetilde{v}_{h}(\cdot, 0)=\widetilde{v}_{0} \in \mathbf{L}^{2}(\Omega), \\
& \widetilde{T}_{h}(\cdot, 0)=\widetilde{T}_{0} \in H_{0}^{1}(\Omega), \frac{\partial \widetilde{T}_{h}}{\partial t}(\cdot, 0)=\widetilde{T}_{1} \in L^{2}(\Omega)
\end{aligned}
$$

where $a_{h}, \Psi_{h}, c_{h}, \phi_{h}$ and $K_{h}$ are given by

$$
\begin{aligned}
a_{h} \quad: \quad L^{2}\left(0, \tau ; \mathbf{H}^{1}(\Omega)\right) \times L^{2}\left(0, \tau ; \mathbf{H}^{1}(\Omega)\right) \rightarrow \mathbb{R} \\
(u, v) \mapsto \sum_{n=0}^{N-1} \int_{t_{n}}^{t_{n+1}} \int_{\Omega} 2 \mu_{n} D_{i j}(u) D_{i j}(v) d x d t
\end{aligned}
$$




$$
\begin{aligned}
\Psi_{h}: & L^{2}\left(0, \tau ; \mathbf{L}^{2}\left(\Gamma_{0}\right)\right) \rightarrow \mathbb{R} \\
u & \mapsto \sum_{n=0}^{N-1} \int_{t_{n}}^{t_{n+1}} \int_{\Gamma_{0}} \ell_{n}|u| d x_{1} d t
\end{aligned}
$$

and

$c_{h}(\cdot, t)=c_{n}(\cdot, t), \phi_{h}(\cdot, t)=\phi_{n}(\cdot, t), K_{h}(\cdot, t)=K_{n}(\cdot) \quad \forall t \in\left[t_{n}, t_{n+1}\right), \forall n \in\{0, \ldots, N-1\}$.

Let us observe that $a_{h}$ and $\Psi_{h}$ fit the assumptions of Theorem $6.2 \mathrm{in}^{29}$ and Proposition $3.2 \mathrm{in}^{30}$. Hence the following problem

Find

$$
\widetilde{v} \in L^{2}\left(0, \tau ; \mathcal{V}_{0 \text { div }}\right) \cap L^{\infty}\left(0, \tau ; \mathbf{L}^{2}(\Omega)\right), p \in H^{-1}\left(0, \tau ; L_{0}^{2}(\Omega)\right)
$$

such that, for all $\varphi \in \mathcal{V}_{0}$ and $\chi \in \mathcal{D}(0, \tau)$, we have

$$
\begin{aligned}
& \left\langle\frac{d}{d t}(\widetilde{v}, \varphi)+b(\widetilde{v}, \widetilde{v}, \varphi), \chi\right\rangle_{\mathcal{D}^{\prime}(0, \tau), \mathcal{D}(0, \tau)}+a_{h}(\widetilde{v}, \varphi \chi) \\
& -\left\langle\int_{\Omega} \operatorname{pdiv}(\varphi) d x, \chi\right\rangle_{\mathcal{D}^{\prime}(0, \tau), \mathcal{D}(0, \tau)}+\Psi_{h}(\widetilde{v}+\varphi \chi)-\Psi_{h}(\widetilde{v}) \\
& \geq\left\langle\left(f-G_{0} \frac{\partial \zeta}{\partial t}, \varphi\right), \chi\right\rangle_{\mathcal{D}^{\prime}(0, \tau), \mathcal{D}(0, \tau)}-a_{h}\left(G_{0} \zeta, \varphi \chi\right) \\
& -\left\langle b\left(G_{0} \zeta, \widetilde{v}+G_{0} \zeta, \varphi\right)+b\left(\widetilde{v}, G_{0} \zeta, \varphi\right), \chi\right\rangle_{\mathcal{D}^{\prime}(0, \tau), \mathcal{D}(0, \tau)}
\end{aligned}
$$

with the initial condition

$$
\widetilde{v}(\cdot, 0)=\widetilde{v}_{0} \in \mathbf{L}^{2}(\Omega)
$$

admits an unique solution $(\widetilde{v}, p)$ and $\frac{\partial \widetilde{v}}{\partial t} \in L^{2}\left(0, \tau ;\left(\mathcal{V}_{0 d i v}\right)^{\prime}\right)$. With the same computations as in Proposition 3.2 in $^{30}$, we infer that $\widetilde{v}=\widetilde{v}_{h}$. Let us define now $p_{h} \in H^{-1}\left(0, \tau ; L_{0}^{2}(\Omega)\right)$ by $p_{h}=p$.

Lemma 3.4. Assume that (6)-(12), (15) and (16) hold. Then, for all $n \in\{0, \ldots, N-1\}$, the restriction of $p_{h}$ on $\left(t_{n}, t_{n+1}\right)$ coïncide with $p_{h}^{n}$.

Proof. We infer from (19) that

$$
\begin{aligned}
& \left\langle\frac{d}{d t}\left(\widetilde{v}_{h}, \varphi\right)+b\left(\widetilde{v}_{h}, \widetilde{v}_{h}, \varphi\right), \chi\right\rangle_{\mathcal{D}^{\prime}(0, \tau), \mathcal{D}(0, \tau)}+a_{h}\left(\widetilde{v}_{h}, \varphi \chi\right) \\
& -\left\langle\int_{\Omega} p_{h} \operatorname{div}(\varphi) d x, \chi\right\rangle_{\mathcal{D}^{\prime}(0, \tau), \mathcal{D}(0, \tau)}=\left\langle\left(f-G_{0} \frac{\partial \zeta}{\partial t}, \varphi\right), \chi\right\rangle_{\mathcal{D}^{\prime}(0, \tau), \mathcal{D}(0, \tau)}-a_{h}\left(G_{0} \zeta, \varphi \chi\right) \\
& -\left\langle b\left(G_{0} \zeta, \widetilde{v}+G_{0} \zeta, \varphi\right)+b\left(\widetilde{v}, G_{0} \zeta, \varphi\right), \chi\right\rangle_{\mathcal{D}^{\prime}(0, \tau), \mathcal{D}(0, \tau)}
\end{aligned}
$$


for any $\varphi \in \mathbf{H}_{0}^{1}(\Omega)$ and for any $\chi \in \mathcal{D}(0, \tau)$. Now let $n \in\{0, \ldots, N-1\}$ and $\chi \in \mathcal{D}\left(t_{n}, t_{n+1}\right)$ extended by 0 to $(0, \tau)$. With $\left(\mathrm{P}_{\mathrm{h} \text { flow }}^{\mathrm{n}}\right)$ we have

$$
\begin{aligned}
& \left\langle\frac{d}{d t}\left(\widetilde{v}_{h}^{n}, \varphi\right)+b\left(\widetilde{v}_{h}^{n}, \widetilde{v}_{h}^{n}, \varphi\right), \chi\right\rangle_{\mathcal{D}^{\prime}\left(t_{n}, t_{n+1}\right), \mathcal{D}\left(t_{n}, t_{n+1}\right)}+a_{n}\left(\widetilde{v}_{h}^{n}, \varphi \chi\right) \\
& -\left\langle\int_{\Omega} p_{h}^{n} \operatorname{div}(\varphi) d x, \chi\right\rangle_{\mathcal{D}^{\prime}\left(t_{n}, t_{n+1}\right), \mathcal{D}\left(t_{n}, t_{n+1}\right)}=\left\langle\left(f-G_{0} \frac{\partial \zeta}{\partial t}, \varphi\right), \chi\right\rangle_{\mathcal{D}^{\prime}\left(t_{n}, t_{n+1}\right), \mathcal{D}\left(t_{n}, t_{n+1}\right)}-a_{n}\left(G_{0} \zeta, \varphi \chi\right) \\
& -\left\langle b\left(G_{0} \zeta, \widetilde{v}_{h}^{n}+G_{0} \zeta, \varphi\right)+b\left(\widetilde{v}_{h}^{n}, G_{0} \zeta, \varphi\right), \chi\right\rangle_{\mathcal{D}^{\prime}\left(t_{n}, t_{n+1}\right), \mathcal{D}\left(t_{n}, t_{n+1}\right)} .
\end{aligned}
$$

Since $\widetilde{v}_{h}(\cdot, t)=\widetilde{v}_{h}^{n}(\cdot, t)$ for all $t \in\left[t_{n}, t_{n+1}\right]$ we obtain

$$
\left\langle\int_{\Omega}\left(p_{h}-p_{h}^{n}\right) \operatorname{div}(\varphi) d x, \chi\right\rangle_{\mathcal{D}^{\prime}\left(t_{n}, t_{n+1}\right), \mathcal{D}\left(t_{n}, t_{n+1}\right)}=0
$$

for any $\varphi \in \mathbf{H}_{0}^{1}(\Omega)$ and for any $\chi \in \mathcal{D}\left(t_{n}, t_{n+1}\right)$, where we have identified $p_{h}$ with its restriction on $\left(t_{n}, t_{n+1}\right)$. But, for any $w \in L_{0}^{2}(\Omega)$ there exists $\varphi \in \mathbf{H}_{0}^{1}(\Omega)$ such that $\operatorname{div}(\varphi)=w\left({ }^{33}\right)$. Thus

$$
\left\langle\int_{\Omega}\left(p_{h}-p_{h}^{n}\right) w d x, \chi\right\rangle_{\mathcal{D}^{\prime}\left(t_{n}, t_{n+1}\right), \mathcal{D}\left(t_{n}, t_{n+1}\right)}=0
$$

for any $w \in L_{0}^{2}(\Omega)$ and for any $\chi \in \mathcal{D}\left(t_{n}, t_{n+1}\right)$. It follows that, for any $\widetilde{w} \in L^{2}(\Omega)$ and for any $\chi \in \mathcal{D}\left(t_{n}, t_{n+1}\right)$, we have

$$
\left\langle\int_{\Omega}\left(p_{h}-p_{h}^{n}\right) \widetilde{w} d x, \chi\right\rangle_{\mathcal{D}^{\prime}\left(t_{n}, t_{n+1}\right), \mathcal{D}\left(t_{n}, t_{n+1}\right)}=\left\langle\int_{\Omega}\left(p_{h}-p_{h}^{n}\right) w d x, \chi\right\rangle_{\mathcal{D}^{\prime}\left(t_{n}, t_{n+1}\right), \mathcal{D}\left(t_{n}, t_{n+1}\right)}=0
$$

with

$$
w=\widetilde{w}-\frac{1}{\operatorname{meas}(\Omega)} \int_{\Omega} \widetilde{w}
$$

By density of $\mathcal{D}\left(t_{n}, t_{n+1}\right) \otimes L^{2}(\Omega)$ into $H_{0}^{1}\left(t_{n}, t_{n+1} ; L^{2}(\Omega)\right)$, we get

$$
\left\langle p_{h}-p_{h}^{n}, \eta\right\rangle_{H^{-1}\left(t_{n}, t_{n+1} ; L^{2}(\Omega)\right), H_{0}^{1}\left(t_{n}, t_{n+1} ; L^{2}(\Omega)\right)}=0
$$

for any $\eta \in H_{0}^{1}\left(t_{n}, t_{n+1} ; L^{2}(\Omega)\right)$ which implies that the restriction of $p_{h}$ on $\left(t_{n}, t_{n+1}\right)$ coïncide with $p_{h}^{n}$.

\section{Convergence}

In order to pass to the limit as $N$ tends to $+\infty$ (i.e. as $h$ tends to zero), we have to establish some a priori estimates for $\widetilde{v}_{h}, p_{h}$ and $\widetilde{T}_{h}$.

By reminding Proposition 3.2 in $^{30}$, and Lemmas 4.1 and 5.1 in $^{29}$ (let us emphasize that the estimates do not depend on the temperature field nor on the Tresca's threshold), we get 
Proposition 4.1. Under the assumptions (6)-(12), (15) and (16), there exists a constant $C$, independent of $h$, such that

$$
\max \left(\left\|\widetilde{v}_{h}\right\|_{L^{\infty}\left(0, \tau ; \mathbf{L}^{2}(\Omega)\right)},\left\|\widetilde{v}_{h}\right\|_{L^{2}\left(0, \tau ; \mathbf{H}^{1}(\Omega)\right)}\right) \leq C
$$

and

$$
\left\|p_{h}\right\|_{H^{-1}\left(0, \tau ; L^{2}(\Omega)\right)} \leq C
$$

Next we observe that, for all $\varphi \in \mathcal{V}_{0 \text { div }}$ and for all $\chi \in \mathcal{D}(0, \tau)$, we have

$$
\begin{aligned}
& \left|\Psi_{h}\left(\widetilde{v}_{h}\right)-\Psi_{h}\left(\widetilde{v}_{h} \pm \varphi \chi\right)\right| \leq \sum_{n=0}^{N-1} \int_{t_{n}}^{t_{n+1}} \int_{\Gamma_{0}} \ell_{n}\|\varphi \chi\| d x_{1} d t \leq \sum_{n=0}^{N-1} \int_{t_{n}}^{t_{n+1}}\left\|\ell\left(\cdot, t_{n}\right)\right\|_{\mathbf{L}^{2}\left(\Gamma_{0}\right)}\|\varphi\|_{L^{2}\left(\Gamma_{0}\right)}|\chi| d t \\
& \leq \gamma(\Omega) \sqrt{\tau}\|\ell\|_{C^{0}\left([0, \tau] ; L^{2}\left(\Gamma_{0}\right)\right)}\|\varphi \chi\|_{L^{2}\left(0, \tau ; \mathbf{H}^{1}(\Omega)\right)}
\end{aligned}
$$

where $\gamma(\Omega)$ is the norm of the trace operator from $\mathbf{H}^{1}(\Omega)$ into $\mathbf{L}^{2}(\partial \Omega)$. Then, by reproducing the same computations as in Lemma 3.1 in $^{30}$, we obtain

Proposition 4.2. Under the assumptions (6)-(12), (15) and (16), there exists a constant $C^{\prime}$, independent of $h$, such that

$$
\left\|\frac{\partial \widetilde{v}_{h}}{\partial t}\right\|_{L^{2}\left(0, \tau ;\left(\mathcal{V}_{0 d i v}\right)^{\prime}\right)} \leq C^{\prime}
$$

We may also obtain some a priori estimates for $\widetilde{T}_{h}$.

Proposition 4.3. Let us assume that (6)-(12) and (16) hold. Then there exists $N_{*} \in \mathbb{N}^{*}$ and a constant $C^{\prime \prime}$, independent of $h$, such that, for all $N \geq N_{*}$

$$
\max \left(\left\|\widetilde{T}_{h}\right\|_{L^{\infty}\left(0, \tau ; H_{0}^{1}(\Omega)\right)},\left\|\frac{\partial \widetilde{T}_{h}}{\partial t}\right\|_{L^{\infty}\left(0, \tau ; L^{2}(\Omega)\right)},\left\|\frac{\partial^{2} \widetilde{T}_{h}}{\partial t^{2}}\right\|_{L^{2}\left(0, \tau ; H^{-1}(\Omega)\right)}\right) \leq C^{\prime \prime} .
$$

Proof. Let $N \geq\left\lfloor\frac{c_{*}^{2} \tau \exp (\tau)}{d^{2}}\right\rfloor+1$ and $n \in\{0, \ldots, N-1\}$. With (14), we get the following equality

$$
\begin{aligned}
& d\left\|\frac{\partial \widetilde{T}_{h}^{n}}{\partial t}(\cdot, s)\right\|_{L^{2}(\Omega)}^{2}+2 \int_{t_{n}}^{s} \int_{\Omega} c_{n}\left(\frac{\partial \widetilde{T}_{h}^{n}}{\partial t}\right)^{2} d x d t+\left(K_{n} \nabla \widetilde{T}_{h}^{n}(\cdot, s), \nabla \widetilde{T}_{h}^{n}(\cdot, s)\right) \\
& =d\left\|\frac{\partial \widetilde{T}_{h}^{n}}{\partial t}\left(\cdot, t_{n}\right)\right\|_{L^{2}(\Omega)}^{2}+\left(K_{n} \nabla \widetilde{T}_{h}^{n}\left(\cdot, t_{n}\right), \nabla \widetilde{T}_{h}^{n}\left(\cdot, t_{n}\right)\right) \\
& +2 \int_{t_{n}}^{s} \int_{\Omega}\left(\phi_{n}-d \frac{\partial^{2} G}{\partial t^{2}}-c_{n} \frac{\partial G}{\partial t}\right) \frac{\partial \widetilde{T}_{h}^{n}}{\partial t} d x d t+2 \int_{t_{n}}^{s} \int_{\Omega} \operatorname{div}\left(K_{n} \nabla G\right) \frac{\partial \widetilde{T}_{h}^{n}}{\partial t} d x d t
\end{aligned}
$$


for all $s \in\left[t_{n}, t_{n+1}\right]$. We rewrite the last term of the previous equality as follows:

$$
\begin{aligned}
& \int_{t_{n}}^{s} \int_{\Omega} \operatorname{div}\left(K_{n} \nabla G\right) \frac{\partial \widetilde{T}_{h}^{n}}{\partial t} d x d t \\
& =-\int_{t_{n}}^{s} \int_{\Omega} \operatorname{div}\left(K_{n} \nabla\left(\frac{\partial G}{\partial t}\right)\right) \widetilde{T}_{h}^{n} d x d t+\int_{\Omega} \operatorname{div}\left(K_{n} \nabla(G(\cdot, s))\right) \widetilde{T}_{h}^{n}(\cdot, s) d x \\
& -\int_{\Omega} \operatorname{div}\left(K_{n}\left(\nabla G\left(\cdot, t_{n}\right)\right)\right) \widetilde{T}_{h}^{n}\left(\cdot, t_{n}\right) d x=\int_{t_{n}}^{s}\left(K_{n} \nabla\left(\frac{\partial G}{\partial t}\right)(\cdot, t), \nabla \widetilde{T}_{h}^{n}(\cdot, t)\right) d t \\
& -\left(K_{n}(\nabla G(\cdot, s)), \nabla\left(\widetilde{T}_{h}^{n}(\cdot, s)\right)\right)+\left(K_{n} \nabla\left(G\left(\cdot, t_{n}\right)\right), \nabla\left(\widetilde{T}_{h}^{n}\left(\cdot, t_{n}\right)\right)\right) .
\end{aligned}
$$

With assumption (10) and Young's inequality, we get

$$
\begin{aligned}
& d\left\|\frac{\partial \widetilde{T}_{h}^{n}}{\partial t}(\cdot, s)\right\|_{L^{2}(\Omega)}^{2}+c^{*} \int_{t_{n}}^{s}\left\|\frac{\partial \widetilde{T}_{h}^{n}}{\partial t}(\cdot, t)\right\|_{L^{2}(\Omega)}^{2} d t+\left(K_{n} \nabla \widetilde{T}_{h}^{n}(\cdot, s), \nabla\left(\widetilde{T}_{h}^{n}(\cdot, s)+2 G(\cdot, s)\right)\right) \\
& \leq d\left\|\frac{\partial \widetilde{T}_{h}^{n}}{\partial t}\left(\cdot, t_{n}\right)\right\|_{L^{2}(\Omega)}^{2}+\left(K_{n} \nabla \widetilde{T}_{h}^{n}\left(\cdot, t_{n}\right), \nabla\left(\widetilde{T}_{h}^{n}\left(\cdot, t_{n}\right)+2 G\left(\cdot, t_{n}\right)\right)\right) \\
& +\frac{3}{c^{*}}\left\|\phi_{h}\right\|_{L^{2}\left(t_{n}, t_{n+1} ; L^{2}(\Omega)\right)}^{2}+\frac{3 d^{2}}{c^{*}}\left\|\frac{\partial^{2} G}{\partial t^{2}}\right\|_{L^{2}\left(t_{n}, t_{n+1} ; L^{2}(\Omega)\right)}^{2}+\frac{3 c_{*}^{2}}{c^{*}}\left\|\frac{\partial G}{\partial t}\right\|_{L^{2}\left(t_{n}, t_{n+1} ; L^{2}(\Omega)\right)}^{2} \\
& +2 \int_{t_{n}}^{s}\left(K_{n} \nabla\left(\frac{\partial G}{\partial t}\right)(\cdot, t), \nabla \widetilde{T}_{h}^{n}(\cdot, t)\right) d t
\end{aligned}
$$

which yields

$$
\begin{aligned}
& d\left\|\frac{\partial \widetilde{T}_{h}^{n}}{\partial t}(\cdot, s)\right\|_{L^{2}(\Omega)}^{2}+c^{*} \int_{t_{n}}^{s}\left\|\frac{\partial \widetilde{T}_{h}^{n}}{\partial t}(\cdot, t)\right\|_{L^{2}(\Omega)}^{2} d t+\left(K_{n} \nabla\left(\widetilde{T}_{h}^{n}(\cdot, s)+G(\cdot, s)\right), \nabla\left(\widetilde{T}_{h}^{n}(\cdot, s)+G(\cdot, s)\right)\right) \\
& \leq d\left\|\frac{\partial \widetilde{T}_{h}^{n}}{\partial t}\left(\cdot, t_{n}\right)\right\|_{L^{2}(\Omega)}^{2}+\left(K_{n} \nabla\left(\widetilde{T}_{h}^{n}\left(\cdot, t_{n}\right)+G\left(\cdot, t_{n}\right)\right), \nabla\left(\widetilde{T}_{h}^{n}\left(\cdot, t_{n}\right)+G\left(\cdot, t_{n}\right)\right)\right) \\
& +\frac{3}{c^{*}}\left\|\phi_{h}\right\|_{L^{2}\left(t_{n}, t_{n+1} ; L^{2}(\Omega)\right)}^{2}+\frac{3 d^{2}}{c^{*}}\left\|\frac{\partial^{2} G}{\partial t^{2}}\right\|_{L^{2}\left(t_{n}, t_{n+1} ; L^{2}(\Omega)\right)}^{2}+\frac{3 c_{*}^{2}}{c^{*}}\left\|\frac{\partial G}{\partial t}\right\|_{L^{2}\left(t_{n}, t_{n+1} ; L^{2}(\Omega)\right)}^{2} \\
& +2 \int_{t_{n}}^{s}\left(K_{n} \nabla\left(\frac{\partial G}{\partial t}\right)(\cdot, t), \nabla\left(\widetilde{T}_{h}^{n}(\cdot, t)+G(\cdot, t)\right)\right) d t \\
& \leq d\left\|\frac{\partial \widetilde{T}_{h}^{n}}{\partial t}\left(\cdot, t_{n}\right)\right\|_{L^{2}(\Omega)}^{2}+\left(K_{n} \nabla\left(\widetilde{T}_{h}^{n}\left(\cdot, t_{n}\right)+G\left(\cdot, t_{n}\right)\right), \nabla\left(\widetilde{T}_{h}^{n}\left(\cdot, t_{n}\right)+G\left(\cdot, t_{n}\right)\right)\right) \\
& +\frac{3}{c^{*}}\left\|\phi_{h}\right\|_{L^{2}\left(t_{n}, t_{n+1} ; L^{2}(\Omega)\right)}^{2}+\frac{3 d^{2}}{c^{*}}\left\|\frac{\partial^{2} G}{\partial t^{2}}\right\|_{L^{2}\left(t_{n}, t_{n+1} ; L^{2}(\Omega)\right)}^{2}+\frac{3 c_{*}^{2}}{c^{*}}\left\|\frac{\partial G}{\partial t}\right\|_{L^{2}\left(t_{n}, t_{n+1} ; L^{2}(\Omega)\right)}^{2} \\
& +\|K\|_{C^{0}\left([0, \tau] ;\left(L^{\infty}(\Omega)\right)^{4}\right)}^{2}\left\|\nabla\left(\frac{\partial G}{\partial t}\right)\right\|_{L^{2}\left(t_{n}, t_{n+1} ; \mathbf{L}^{2}(\Omega)\right)}^{2}+\int_{t_{n}}\left\|\nabla\left(\widetilde{T}_{h}^{n}(\cdot, t)+G(\cdot, t)\right)\right\|_{\mathbf{L}^{2}(\Omega)}^{2} d t .
\end{aligned}
$$


Let $z_{n}=x_{n}+y_{n}$ with

$$
\begin{aligned}
& x_{n}=\frac{3}{c^{*}}\left\|\phi_{h}\right\|_{L^{2}\left(t_{n}, t_{n+1} ; L^{2}(\Omega)\right)}^{2}+\frac{3 d^{2}}{c^{*}}\left\|\frac{\partial^{2} G}{\partial t^{2}}\right\|_{L^{2}\left(t_{n}, t_{n+1} ; L^{2}(\Omega)\right)}^{2}+\frac{3 c_{*}^{2}}{c^{*}}\left\|\frac{\partial G}{\partial t}\right\|_{L^{2}\left(t_{n}, t_{n+1} ; L^{2}(\Omega)\right)}^{2} \\
& +\|K\|_{C^{0}\left([0, \tau] ;\left(L^{\infty}(\Omega)\right)^{4}\right)}^{2}\left\|\nabla\left(\frac{\partial G}{\partial t}\right)\right\|_{L^{2}\left(t_{n}, t_{n+1} ; \mathbf{L}^{2}(\Omega)\right)}^{2}
\end{aligned}
$$

and

$$
y_{n}=d\left\|\frac{\partial \widetilde{T}_{h}^{n}}{\partial t}\left(\cdot, t_{n}\right)\right\|_{L^{2}(\Omega)}^{2}+\left(K_{n} \nabla\left(\widetilde{T}_{h}^{n}\left(\cdot, t_{n}\right)+G\left(\cdot, t_{n}\right)\right), \nabla\left(\widetilde{T}_{h}^{n}\left(\cdot, t_{n}\right)+G\left(\cdot, t_{n}\right)\right)\right)
$$

for all $n \in\{0, \ldots, N-1\}$. With (9) we infer that

$$
\left\|\nabla\left(\widetilde{T}_{h}^{n}(\cdot, s)+G(\cdot, s)\right)\right\|_{L^{2}(\Omega)}^{2} \leq \frac{1}{\alpha^{*}}\left(x_{n}+y_{n}\right)+\frac{1}{\alpha^{*}} \int_{t_{n}}^{s}\left\|\nabla\left(\widetilde{T}_{h}^{n}(\cdot, t)+G(\cdot, t)\right)\right\|_{\mathbf{L}^{2}(\Omega)}^{2} d t
$$

and Grönwall's lemma implies that

$$
\left\|\nabla\left(\widetilde{T}_{h}^{n}(\cdot, s)+G(\cdot, s)\right)\right\|_{L^{2}(\Omega)}^{2} \leq \frac{1}{\alpha^{*}} z_{n} \exp \left(\frac{s-t_{n}}{\alpha^{*}}\right)
$$

for all $s \in\left[t_{n}, t_{n+1}\right]$. By replacing in (24), we obtain

$$
\begin{aligned}
& d\left\|\frac{\partial \widetilde{T}_{h}^{n}}{\partial t}(\cdot, s)\right\|_{L^{2}(\Omega)}^{2}+c^{*} \int_{t_{n}}^{s}\left\|\frac{\partial \widetilde{T}_{h}^{n}}{\partial t}(\cdot, t)\right\|_{L^{2}(\Omega)}^{2} d t \\
& +\left(K_{n} \nabla\left(\widetilde{T}_{h}^{n}(\cdot, s)+G(\cdot, s)\right), \nabla\left(\widetilde{T}_{h}^{n}(\cdot, s)+G(\cdot, s)\right)\right) \leq z_{n} \exp \left(\frac{s-t_{n}}{\alpha^{*}}\right)
\end{aligned}
$$

for all $s \in\left[t_{n}, t_{n+1}\right]$. With $s=t_{n+1}$ we have

$$
\begin{aligned}
& y_{n+1} \leq z_{n} \exp \left(\frac{t_{n+1}-t_{n}}{\alpha^{*}}\right)+\left(K_{n+1} \nabla\left(\widetilde{T}_{h}^{n}\left(\cdot, t_{n+1}\right)+G\left(\cdot, t_{n+1}\right)\right), \nabla\left(\widetilde{T}_{h}^{n}\left(\cdot, t_{n+1}\right)+G\left(\cdot, t_{n+1}\right)\right)\right) \\
& -\left(K_{n} \nabla\left(\widetilde{T}_{h}^{n}\left(\cdot, t_{n+1}\right)+G\left(\cdot, t_{n+1}\right)\right), \nabla\left(\widetilde{T}_{h}^{n}\left(\cdot, t_{n+1}\right)+G\left(\cdot, t_{n+1}\right)\right)\right) .
\end{aligned}
$$

With assumption (8) we get

$$
y_{n+1} \leq\left(y_{n}+x_{n}\right) \exp \left(\frac{h}{\alpha^{*}}\right)+\frac{h}{\alpha^{*}}\left\|\frac{\partial K}{\partial t}\right\|_{L^{\infty}\left(t_{n}, t_{n+1} ;\left(L^{\infty}(\Omega)\right)^{4}\right)} y_{n+1} .
$$

Let

$$
N_{*} \geq \max \left(\left\lfloor\frac{c_{*}^{2} \tau \exp (\tau)}{d^{2}}\right\rfloor+1,\left\lfloor\frac{2 \tau}{\alpha^{*}}\left\|\frac{\partial K}{\partial t}\right\|_{L^{\infty}\left(0, \tau ;\left(L^{\infty}(\Omega)\right)^{4}\right)}\right\rfloor+1\right)
$$


and

$$
C=\frac{1}{\alpha^{*}}\left(2\left\|\frac{\partial K}{\partial t}\right\|_{L^{\infty}\left(0, \tau ;\left(L^{\infty}(\Omega)\right)^{4}\right)}+1\right) .
$$

For all $N \geq N_{*}$ and for all $n \in\{0, \ldots, N-1\}$, we have

$$
y_{n+1} \leq\left(y_{n}+x_{n}\right) \exp (C h) .
$$

Hence

$$
y_{n} \leq y_{0} \exp (C n h)+\sum_{k=0}^{n-1} x_{k} \exp (C(n-k) h)
$$

and

$$
z_{n} \leq \exp (C \tau)\left(y_{0}+\sum_{k=0}^{n} x_{k}\right)
$$

for all $n \in\{0, \ldots, N-1\}$. But

$$
\begin{aligned}
& \sum_{k=0}^{n} x_{k} \leq \frac{3}{c^{*}}\left\|\phi_{h}\right\|_{L^{2}\left(0, \tau ; L^{2}(\Omega)\right)}^{2}+\frac{3 d^{2}}{c^{*}}\left\|\frac{\partial^{2} G}{\partial t^{2}}\right\|_{L^{2}\left(0, \tau ; L^{2}(\Omega)\right)}^{2}+\frac{3 c_{*}^{2}}{c^{*}}\left\|\frac{\partial G}{\partial t}\right\|_{L^{2}\left(0, \tau ; L^{2}(\Omega)\right)}^{2} \\
& +\|K\|_{C^{0}\left([0, \tau] ;\left(L^{\infty}(\Omega)\right)^{4}\right)}^{2}\left\|\nabla\left(\frac{\partial G}{\partial t}\right)\right\|_{L^{2}\left(0, \tau ; \mathbf{L}^{2}(\Omega)\right)}^{2}
\end{aligned}
$$

and

$$
\left\|\phi_{h}\right\|_{L^{2}\left(0, \tau ; L^{2}(\Omega)\right)}^{2}=h\left\|\phi\left(\widetilde{v}_{0}+G_{0}\right)\right\|_{L^{2}(\Omega)}^{2}+\int_{h}^{\tau}\left\|\phi\left(\widetilde{v}_{h}(\cdot, t-h)+G_{0} \zeta(t)\right)\right\|_{L^{2}(\Omega)}^{2} d t .
$$

Reminding that $\phi$ is Lipschitz continuous, we may denote by $L_{\phi}$ its Lipschitz constant and we have

$$
\begin{aligned}
& \left\|\phi_{h}\right\|_{L^{2}\left(0, \tau ; L^{2}(\Omega)\right)}^{2} \leq \tau\left\|\phi\left(\widetilde{v}_{0}+G_{0}\right)\right\|_{L^{2}(\Omega)}^{2}+2 \tau|\phi(0)|^{2} \operatorname{meas}(\Omega) \\
& +4 L_{\phi}^{2}\left\|\widetilde{v}_{h}\right\|_{L^{2}\left(0, \tau ; \mathbf{L}^{2}(\Omega)\right)}^{2}+4 L_{\phi}^{2}\left\|G_{0}\right\|_{L^{2}(\Omega)}^{2} \int_{0}^{\tau}|\zeta|^{2} d t .
\end{aligned}
$$

With the results of Proposition 4.1, we infer that $z_{n}$ is bounded by a constant independent of $n$ and $h$. Going back to (25) and (26), and using (18), we get (23)

Now we can pass to the limit. Indeed, with the results of the three previous propositions, we know that there exists a subsequence of $\left(\widetilde{v}_{h}, p_{h}, \widetilde{T}_{h}\right)_{\tau / N_{*}>h>0}$, still denoted $\left(\widetilde{v}_{h}, p_{h}, \widetilde{T}_{h}\right)_{\tau / N_{*}>h>0}$, such that

$$
\begin{aligned}
& \widetilde{v}_{h} \rightarrow \widetilde{v} \text { weakly in } L^{2}\left(0, \tau ; \mathcal{V}_{\text {Odiv }}\right) \\
& \text { and weakly } * \text { in } L^{\infty}\left(0, \tau ; \mathbf{L}^{2}(\Omega)\right),
\end{aligned}
$$




$$
\begin{gathered}
\frac{\partial \widetilde{v}_{h}}{\partial t} \rightarrow \frac{\widetilde{v}}{\partial t} \quad \text { weakly in } L^{2}\left(0, \tau ;\left(\mathcal{V}_{0 d i v}\right)^{\prime}\right), \\
p_{h} \rightarrow p \quad \text { weakly in } H^{-1}\left(0, \tau ; L_{0}^{2}(\Omega)\right),
\end{gathered}
$$

and

$$
\begin{gathered}
\widetilde{T}_{h} \rightarrow \widetilde{T} \quad \text { weakly * in } L^{\infty}\left(0, \tau ; H_{0}^{1}(\Omega)\right), \\
\frac{\partial \widetilde{T}_{h}}{\partial t} \rightarrow \frac{\partial \widetilde{T}}{\partial t} \quad \text { weakly * in } L^{\infty}\left(0, \tau ; L^{2}(\Omega)\right), \\
\frac{\partial^{2} \widetilde{T}_{h}}{\partial t^{2}} \rightarrow \frac{\partial^{2} \widetilde{T}}{\partial t^{2}} \quad \text { weakly in } L^{2}\left(0, \tau ; H^{-1}(\Omega)\right) .
\end{gathered}
$$

Moreover, with Aubin's lemma, we have

$$
\widetilde{v}_{h} \rightarrow \widetilde{v} \quad \text { strongly in } L^{2}\left(0, \tau ; \mathbf{L}^{4}(\Omega) \cap \mathbf{L}^{2}\left(\Gamma_{0}\right)\right)
$$

and with Simon's lemma ${ }^{34}$

$$
\begin{gathered}
\widetilde{v}_{h} \rightarrow \widetilde{v} \quad \text { strongly in } \quad \mathcal{C}^{0}([0, \tau] ; \mathbf{H}), \\
\widetilde{T}_{h} \rightarrow \widetilde{T} \quad \text { strongly in } \mathcal{C}^{0}\left([0, \tau] ; L^{2}(\Omega)\right) \cap \mathcal{C}^{1}([0, \tau] ; H)
\end{gathered}
$$

where $\mathbf{H}$ (resp. $H$ ) is a Banach space such that $\mathbf{L}^{2}(\Omega) \subset \mathbf{H} \subset\left(\mathcal{V}_{\text {odiv }}\right)^{\prime}\left(\right.$ resp. $L^{2}(\Omega) \subset H \subset H^{-1}(\Omega)$ ) with continuous injections and a compact embedding of $\mathbf{L}^{2}(\Omega)$ into $\mathbf{H}$ (resp. of $L^{2}(\Omega)$ into $H$ ). It follows that

$$
\begin{aligned}
& \widetilde{v}(\cdot, 0)=\widetilde{v}_{0}, \\
& \widetilde{T}(\cdot, 0)=\widetilde{T}_{0}, \quad \frac{\partial \widetilde{T}}{\partial t}(\cdot, 0)=\widetilde{T}_{1} .
\end{aligned}
$$

Furthermore,

Theorem 4.4. (Convergence of the splitting technique and existence result for $(P)$ ). Under the assumptions (6)-(12) and (16), the limit triplet $(\widetilde{v}, p, \widetilde{T})$ is solution of problem $(P)$.

Proof. Let $\varphi \in \mathcal{V}_{0}$ and $\chi \in \mathcal{D}(0, \tau)$. For all $h \in\left(0, \tau / N_{*}\right)$ we have

$$
\begin{aligned}
& \left\langle\frac{d}{d t}\left(\widetilde{v}_{h}, \varphi\right)+b\left(\widetilde{v}_{h}, \widetilde{v}_{h}, \varphi\right), \chi\right\rangle_{\mathcal{D}^{\prime}(0, \tau), \mathcal{D}(0, \tau)}+a_{h}\left(\widetilde{v}_{h}, \varphi \chi\right) \\
& -\left\langle\int_{\Omega} p_{h} \operatorname{div}(\varphi) d x, \chi\right\rangle_{\mathcal{D}^{\prime}(0, \tau), \mathcal{D}(0, \tau)}+\Psi_{h}\left(\widetilde{v}_{h}+\varphi \chi\right)-\Psi_{h}\left(\widetilde{v}_{h}\right) \\
& \geq\left\langle\left(f-G_{0} \frac{\partial \zeta}{\partial t}, \varphi\right), \chi\right\rangle_{\mathcal{D}^{\prime}(0, \tau), \mathcal{D}(0, \tau)}-a_{h}\left(G_{0} \zeta, \varphi \chi\right) \\
& -\left\langle b\left(G_{0} \zeta, \widetilde{v}_{h}+G_{0} \zeta, \varphi\right)+b\left(\widetilde{v}_{h}, G_{0} \zeta, \varphi\right), \chi\right\rangle_{\mathcal{D}^{\prime}(0, \tau), \mathcal{D}(0, \tau)} .
\end{aligned}
$$


Reminding that $\ell \in W^{1,2}\left(0, \tau ; L_{+}^{2}\left(\Gamma_{0}\right)\right)$, we obtain

$$
\begin{aligned}
& \left|\Psi_{h}\left(\widetilde{v}_{h}+\varphi \chi\right)-\Psi\left(\widetilde{v}_{h}+\varphi \chi\right)\right|=\left|\sum_{n=0}^{N-1} \int_{t_{n}}^{t_{n+1}} \int_{\Gamma_{0}}\left(\ell_{n}-\ell\right)\right| \widetilde{v}_{h}+\varphi \chi\left|d x_{1} d t\right| \\
& \leq \sum_{n=0}^{N-1} \int_{t_{n}}^{t_{n+1}}\left(\int_{t_{n}}^{t}\left\|\frac{\partial \ell}{\partial t}(\cdot, s)\right\| \|_{L^{2}\left(\Gamma_{0}\right)} d s\right)\left\|\widetilde{v}_{h}(\cdot, t)+\varphi \chi(t)\right\|_{\mathbf{L}^{2}\left(\Gamma_{0}\right)} d t \\
& \leq h\left\|\frac{\partial \ell}{\partial t}(\cdot, s)\right\|_{L^{2}\left(0, \tau ; L^{2}\left(\Gamma_{0}\right)\right)}\left\|\widetilde{v}_{h}+\varphi \chi\right\|_{L^{2}\left(0, \tau ; \mathbf{L}^{2}\left(\Gamma_{0}\right)\right)} .
\end{aligned}
$$

\section{Moreover}

Lemma 4.5. For any $\varphi \in \mathcal{V}_{0}$ and $\chi \in \mathcal{D}(0, \tau)$, we have the following convergences

$$
\begin{aligned}
& a_{h}\left(G_{0} \zeta, \varphi \chi\right) \rightarrow a\left(\widetilde{T}+G ; G_{0} \zeta, \varphi \chi\right), \\
& a_{h}\left(\widetilde{v}_{h}, \varphi \chi\right) \rightarrow a(\widetilde{T}+G ; \widetilde{v}, \varphi \chi) .
\end{aligned}
$$

Proof. Let $\varphi \in \mathcal{V}_{0}$ and $\chi \in \mathcal{D}(0, \tau)$. With the previous convergences we already have

$$
a\left(\widetilde{T}+G ; \widetilde{v}_{h}, \varphi \chi\right) \rightarrow a(\widetilde{T}+G ; \widetilde{v}, \varphi \chi) .
$$

Hence we only need to prove that

$$
\begin{aligned}
& \lim _{h \rightarrow 0}\left|a_{h}\left(G_{0} \zeta, \varphi \chi\right)-a\left(\widetilde{T}+G ; G_{0} \zeta, \varphi \chi\right)\right|=0, \\
& \lim _{h \rightarrow 0}\left|a_{h}\left(\widetilde{v}_{h}, \varphi \chi\right)-a\left(\widetilde{T}+G ; \widetilde{v}_{h}, \varphi \chi\right)\right|=0 .
\end{aligned}
$$

Let us consider first $\varphi \in(\mathcal{D}(\bar{\Omega}))^{2}$. We have

$$
\begin{aligned}
& \left|a_{h}\left(G_{0} \zeta, \varphi \chi\right)-a\left(\widetilde{T}+G ; G_{0} \zeta, \varphi \chi\right)\right| \\
& \leq \sum_{n=0}^{N-1} \int_{t_{n}}^{t_{n+1}} \int_{\Omega} 2\left|\mu_{n}-\mu(\widetilde{T}+G)\right|\left|D_{i j}\left(G_{0} \zeta\right)\right|\left|D_{i j}(\varphi)\right||\chi| d x d t
\end{aligned}
$$

and with assumption (7), we get

$$
\begin{aligned}
& \left|a_{h}\left(G_{0} \zeta, \varphi \chi\right)-a\left(\widetilde{T}+G ; G_{0} \zeta, \varphi \chi\right)\right| \\
& \leq 2 \mu_{*}^{\prime} \sum_{n=0}^{N-1} \int_{t_{n}}^{t_{n+1}}\left(\left\|\widetilde{T}_{h, 0}^{n}-\widetilde{T}\right\|_{L^{2}(\Omega)}+\left\|G\left(\cdot, t_{n}\right)-G(\cdot, t)\right\|_{L^{2}(\Omega)}\right)\left\|G_{0} \zeta\right\|_{\mathbf{H}^{1}(\Omega)}\|\nabla \varphi\|_{L^{\infty}(\Omega)}|\chi| d t .
\end{aligned}
$$

But

$$
\begin{aligned}
& \left\|\widetilde{T}_{h, 0}^{n}-\widetilde{T}\right\|_{L^{2}(\Omega)} \leq\left\|\widetilde{T}_{h}\left(\cdot, t_{n}\right)-\widetilde{T}(\cdot, t)\right\|_{L^{2}(\Omega)} \leq\left\|\int_{t_{n}}^{t} \frac{\partial \widetilde{T}_{h}}{\partial t}(\cdot, s) d s\right\|_{L^{2}(\Omega)}+\left\|\widetilde{T}_{h}-\widetilde{T}\right\|_{\mathcal{C}^{0}\left([0, \tau] ; L^{2}(\Omega)\right)} \\
& \leq \sqrt{t-t_{n}}\left\|\frac{\partial \widetilde{T}_{h}}{\partial t}\right\|_{L^{2}\left(0, \tau ; L^{2}(\Omega)\right)}+\left\|\widetilde{T}_{h}-\widetilde{T}\right\|_{\mathcal{C}^{0}\left([0, \tau] ; L^{2}(\Omega)\right)}
\end{aligned}
$$


and

$$
\left\|G\left(\cdot, t_{n}\right)-G(\cdot, t)\right\|_{L^{2}(\Omega)} \leq \sqrt{t-t_{n}}\left\|\frac{\partial G}{\partial t}\right\|_{L^{2}\left(0, \tau ; L^{2}(\Omega)\right)}
$$

Then

$$
\begin{aligned}
& \left|a_{h}\left(G_{0} \zeta, \varphi \chi\right)-a\left(\widetilde{T}+G ; G_{0} \zeta, \varphi \chi\right)\right| \\
& \leq 2 \mu_{*}^{\prime}\left(\sqrt{h}\left\|\frac{\partial \widetilde{T}_{h}}{\partial t}\right\|_{L^{2}\left(0, \tau ; L^{2}(\Omega)\right)}+\left\|\widetilde{T}_{h}-\widetilde{T}\right\|_{\mathcal{C}^{0}\left([0, \tau] ; L^{2}(\Omega)\right)}\right. \\
& \left.+\sqrt{h}\left\|\frac{\partial G}{\partial t}\right\| \|_{L^{2}\left(0, \tau ; L^{2}(\Omega)\right)}\right) \times \int_{0}^{\tau}\left\|G_{0} \zeta\right\|_{\mathbf{H}^{1}(\Omega)}\|\nabla \varphi\|_{L^{\infty}(\Omega)}|\chi| d t .
\end{aligned}
$$

Similarly

$$
\begin{aligned}
& \left|a_{h}\left(\widetilde{v}_{h}, \varphi \chi\right)-a\left(\widetilde{T}+G ; \widetilde{v}_{h}, \varphi \chi\right)\right| \\
& \leq 2 \mu_{*}^{\prime}\left(\sqrt{h}\left\|\frac{\partial \widetilde{T}_{h}}{\partial t}\right\|_{L^{2}\left(0, \tau ; L^{2}(\Omega)\right)}+\left\|\widetilde{T}_{h}-\widetilde{T}\right\|_{\mathcal{C}^{0}\left([0, \tau] ; L^{2}(\Omega)\right)}\right) \times \int_{0}^{\tau}\left\|\widetilde{v}_{h}\right\|_{\mathbf{H}^{1}(\Omega)}\|\nabla \varphi\|_{L^{\infty}(\Omega)}|\chi| d t . \\
& +\sqrt{h}\left\|\frac{\partial G}{\partial t}\right\|_{L^{2}\left(0, \tau ; L^{2}(\Omega)\right)}
\end{aligned}
$$

With the estimates (20)-(23) and the convergence (35) we infer that

$$
\begin{aligned}
& \lim _{h \rightarrow 0}\left|a_{h}\left(G_{0} \zeta, \varphi \chi\right)-a\left(\widetilde{T}+G ; G_{0} \zeta, \varphi \chi\right)\right|=0, \\
& \lim _{h \rightarrow 0}\left|a_{h}\left(\widetilde{v}_{h}, \varphi \chi\right)-a\left(\widetilde{T}+G ; \widetilde{v}_{h}, \varphi \chi\right)\right|=0
\end{aligned}
$$

for all $\varphi \in(\mathcal{D}(\bar{\Omega}))^{2}$ and for all $\chi \in \mathcal{D}(0, \tau)$. Finally the density of $(\mathcal{D}(\bar{\Omega}))^{2}$ into $\mathbf{H}^{1}(\Omega)$ allows us to conclude.

Now we can pass to the limit in all the terms of (36) and we get

$$
\begin{aligned}
& \left\langle\frac{d}{d t}(\widetilde{v}, \varphi)+b(\widetilde{v}, \widetilde{v}, \varphi), \chi\right\rangle_{\mathcal{D}^{\prime}(0, \tau), \mathcal{D}(0, \tau)}+a(\widetilde{T}+G ; \widetilde{v}, \varphi \chi) \\
& -\left\langle\int_{\Omega} p \operatorname{div}(\varphi) d x, \chi\right\rangle_{\mathcal{D}^{\prime}(0, \tau), \mathcal{D}(0, \tau)}+\Psi(\widetilde{v}+\varphi \chi)-\Psi(\widetilde{v}) \\
& \geq\left\langle\left(f-G_{0} \frac{\partial \zeta}{\partial t}, \varphi\right), \chi\right\rangle_{\mathcal{D}^{\prime}(0, \tau), \mathcal{D}(0, \tau)}-a\left(\widetilde{T}+G ; G_{0} \zeta, \varphi \chi\right) \\
& -\left\langle b\left(G_{0} \zeta, \widetilde{v}+G_{0} \zeta, \varphi\right)+b\left(\widetilde{v}, G_{0} \zeta, \varphi\right), \chi\right\rangle_{\mathcal{D}^{\prime}(0, \tau), \mathcal{D}(0, \tau)}
\end{aligned}
$$

for all $\varphi \in \mathcal{V}_{0}$ and $\chi \in \mathcal{D}(0, \tau)$. 
In order to pass to the limit in the approximate heat problem, we consider a test-function $w=\omega \xi$ with $\omega \in \mathcal{D}(\Omega)$ and $\xi \in \mathcal{D}(0, \tau)$. We have

$$
\begin{aligned}
& d \int_{0}^{\tau}\left\langle\frac{\partial^{2} \widetilde{T}_{h}}{\partial t^{2}}, \omega \xi\right\rangle_{H^{-1}(\Omega), H_{0}^{1}(\Omega)} d t+\int_{0}^{\tau} \int_{\Omega} c_{h} \frac{\partial \widetilde{T}_{h}}{\partial t} \omega \xi d x d t \\
& +\sum_{n=0}^{N-1} \int_{t_{n}}^{t_{n+1}}\left(\left(K_{n}-K\right) \nabla \widetilde{T}_{h}, \nabla \omega\right) \xi d t+\int_{0}^{\tau}\left(K \nabla \widetilde{T}_{h}, \nabla \omega\right) \xi d t \\
& =\int_{0}^{\tau} \int_{\Omega} \phi_{h} \omega \xi d x d t-d \int_{0}^{\tau} \int_{\Omega} \frac{\partial^{2} G}{\partial t^{2}} \omega \xi d x d t-\int_{0}^{\tau} \int_{\Omega} c_{h} \frac{\partial G}{\partial t} \omega \xi d x d t \\
& -\sum_{n=0}^{N-1} \int_{t_{n}}^{t_{n+1}}\left(\left(K_{n}-K\right) \nabla G, \nabla \omega\right) \xi d t-\int_{0}^{\tau}(K \nabla G, \nabla \omega) \xi d t .
\end{aligned}
$$

Reminding that $K_{i j} \in W^{1, \infty}\left(0, \tau ; W^{1, \infty}(\Omega)\right)$ for all $i, j \in\{1,2\}$, we infer that

$$
\begin{aligned}
& \left|\sum_{n=0}^{N-1} \int_{t_{n}}^{t_{n+1}}\left(\left(K_{n}-K\right) \nabla \widetilde{T}_{h}, \nabla \omega\right) \xi d t\right| \\
& \leq\|\xi\|_{L^{\infty}(0, \tau)}\|\nabla \omega\|_{\mathbf{L}^{2}(\Omega)} \sum_{n=0}^{N-1} \int_{t_{n}}^{t_{n+1}}\left\|K\left(\cdot, t_{n}\right)-K(\cdot, t)\right\|_{\left(L^{\infty}(\Omega)\right)^{4}}\left\|\nabla \widetilde{T}_{h}(\cdot, t)\right\|_{\mathbf{L}^{2}(\Omega)} d t \\
& \leq\|\xi\|_{L^{\infty}(0, \tau)}\|\omega\|_{H^{1}(\Omega)} \sum_{n=0}^{N-1} \int_{t_{n}}^{t_{n+1}}\left(\int_{t_{n}}^{t}\left\|\frac{\partial K}{\partial t}(\cdot, s)\right\|_{\left(L^{\infty}(\Omega)\right)^{4}} d s\right)\left\|\widetilde{T}_{h}(\cdot, t)\right\|_{H^{1}(\Omega)} d t \\
& \leq h \sqrt{\tau}\|\xi\|_{L^{\infty}(0, \tau)}\|\omega\|_{H^{1}(\Omega)}\left\|\frac{\partial K}{\partial t}\right\|_{L^{\infty}\left(0, \tau ;\left(L^{\infty}(\Omega)\right)^{4}\right)}\left\|\widetilde{T}_{h}\right\|_{L^{2}\left(0, \tau ; H^{1}(\Omega)\right)} .
\end{aligned}
$$

Similarly

$$
\begin{aligned}
& \left|\sum_{n=0}^{N-1} \int_{t_{n}}^{t_{n+1}}\left(\left(K_{n}-K\right) \nabla G, \nabla \omega\right) \xi d t\right| \\
& \leq h \sqrt{\tau}\|\xi\|_{L^{\infty}(0, \tau)}\|\omega\|_{H^{1}(\Omega)}\left\|\frac{\partial K}{\partial t}\right\|_{L^{\infty}\left(0, \tau ;\left(L^{\infty}(\Omega)\right)^{4}\right)}\|G\|_{L^{2}\left(0, \tau ; \mathbf{H}^{1}(\Omega)\right)} .
\end{aligned}
$$

Let us study now the convergence of the coupling terms.

Lemma 4.6. We have

$$
\begin{aligned}
& \phi_{h} \rightarrow \phi\left(\widetilde{v}+G_{0} \zeta\right) \quad \text { strongly in } L^{2}\left(0, \tau ; L^{2}(\Omega)\right), \\
& c_{h} \rightarrow c\left(\widetilde{v}+G_{0} \zeta\right) \quad \text { strongly in } L^{2}\left(0, \tau ; L^{2}(\Omega)\right) .
\end{aligned}
$$

Proof. Let $h \in\left(0, \tau / N_{*}\right)$. By using the Lipschitz continuity of $\phi$, we have

$$
\begin{aligned}
& \int_{0}^{\tau}\left\|\phi_{h}(\cdot, t)-\phi\left(\widetilde{v}(\cdot, t)+G_{0} \zeta(t)\right)\right\|_{L^{2}(\Omega)}^{2} d t \leq 2 L_{\phi}^{2} \int_{0}^{h}\left\|\widetilde{v}_{0}-\widetilde{v}(\cdot, t)\right\|_{\mathbf{L}^{2}(\Omega)}^{2} d t \\
& +2 L_{\phi}^{2} \int_{0}^{h}\left\|G_{0}\right\|_{\mathbf{L}^{2}(\Omega)}^{2}|1-\zeta(t)|^{2} d t+L_{\phi}^{2} \int_{h}^{\tau}\left\|\widetilde{v}_{h}(\cdot, t-h)-\widetilde{v}(\cdot, t)\right\|_{\mathbf{L}^{2}(\Omega)}^{2} d t \\
& \leq 2 L_{\phi}^{2} h\left\|G_{0}\right\|_{\mathbf{L}^{2}(\Omega)}^{2}\|1-\zeta\|_{L^{\infty}(0, \tau)}^{2}+2 L_{\phi}^{2} \sqrt{\operatorname{meas}(\Omega)}\left\|\widetilde{v}_{h}-\widetilde{v}\right\|_{L^{2}\left(0, \tau ; \mathbf{L}^{4}(\Omega)\right)}^{2}+2 L_{\phi}^{2}\left\|w_{h}-\widetilde{v}\right\|_{L^{2}\left(0, \tau ; \mathbf{L}^{2}(\Omega)\right.}^{2}
\end{aligned}
$$


with

$$
\left\{\begin{array}{l}
w_{h}(\cdot, t)=\widetilde{v}_{0} \forall t \in[0, h) \\
w_{h}(\cdot, t)=\widetilde{v}(t-h) \forall t \in[h, \tau]
\end{array}\right.
$$

But

$$
\int_{0}^{\tau}\left\|w_{h}(\cdot, t)\right\|_{\mathbf{L}^{2}(\Omega)}^{2} d t=\int_{0}^{\tau}\|\widetilde{v}(\cdot, t)\|_{\mathbf{L}^{2}(\Omega)}^{2} d t+h\left\|\widetilde{v}_{0}\right\|_{\mathbf{L}^{2}(\Omega)}^{2}-\int_{\tau-h}^{\tau}\|\widetilde{v}(\cdot, t)\|_{\mathbf{L}^{2}(\Omega)}^{2} d t .
$$

Hence $\left(\left\|w_{h}\right\|_{L^{2}\left(0, \tau ; \mathbf{L}^{2}(\Omega)\right)}\right)_{\tau / N_{*}>h>0}$ is bounded independently of $h$ and

$$
\lim _{h \rightarrow 0}\left\|w_{h}\right\|_{L^{2}\left(0, \tau ; \mathbf{L}^{2}(\Omega)\right)}=\|\widetilde{v}\|_{L^{2}\left(0, \tau ; \mathbf{L}^{2}(\Omega)\right)} .
$$

Furthermore, for any $\varphi \in(\mathcal{D}(\Omega))^{2}$ and $\xi \in \mathcal{D}(0, \tau)$, we have

$$
\begin{aligned}
& \int_{0}^{\tau}\left(w_{h}(\cdot, t)-\widetilde{v}(\cdot, t), \varphi\right) \xi(t) d t \\
& =\int_{0}^{h}\left(\widetilde{v}_{0}, \varphi\right) \xi(t) d t+\int_{0}^{\tau-h}(\widetilde{v}(\cdot, t), \varphi)(\xi(t+h)-\xi(t)) d t-\int_{\tau-h}^{\tau}(\widetilde{v}(\cdot, t), \varphi) \xi(t) d t .
\end{aligned}
$$

It follows that

$$
\begin{aligned}
& \left|\int_{0}^{\tau}\left(w_{h}(\cdot, t)-\widetilde{v}(\cdot, t), \varphi\right) \xi(t) d t\right| \leq h\left\|\widetilde{v}_{0}\right\|_{\mathbf{L}^{2}(\Omega)}\|\varphi\|_{\mathbf{L}^{2}(\Omega)}\|\xi\|_{L^{\infty}(0, \tau)} \\
& +h \sqrt{\tau}\|\widetilde{v}\|_{L^{2}\left(0, \tau ; \mathbf{L}^{2}(\Omega)\right)}\|\varphi\|_{\mathbf{L}^{2}(\Omega)}\left\|\frac{\partial \xi}{\partial t}\right\|_{L^{\infty}(0, \tau)}+\sqrt{h}\|\widetilde{v}\|_{L^{2}\left(0, \tau ; \mathbf{L}^{2}(\Omega)\right)}\|\varphi\|_{\mathbf{L}^{2}(\Omega)}\|\xi\|_{L^{\infty}(0, \tau)} .
\end{aligned}
$$

We may conclude that

$$
w_{h} \rightarrow \widetilde{v} \quad \text { strongly in } L^{2}\left(0, \tau ; \mathbf{L}^{2}(\Omega)\right)
$$

Going back to (38), we obtain the first part of the announced result. The second part of the announced result can be proved with similar computations.

We can pass now to the limit in all the terms of (37). We get

$$
\begin{aligned}
& d \int_{0}^{\tau}\left\langle\frac{\partial^{2} \widetilde{T}}{\partial t^{2}}, \omega \xi\right\rangle_{H^{-1}(\Omega), H_{0}^{1}(\Omega)} d t+\int_{0}^{\tau} \int_{\Omega} c\left(\widetilde{v}+G_{0} \zeta\right) \frac{\partial \widetilde{T}}{\partial t} \omega \xi d x d t+\int_{0}^{\tau}(K \nabla \widetilde{T}, \nabla \omega) \xi d t \\
& =\int_{0}^{\tau} \int_{\Omega} \phi\left(\widetilde{v}+G_{0} \zeta\right) \omega \xi d x d t-d \int_{0}^{\tau} \int_{\Omega} \frac{\partial^{2} G}{\partial t^{2}} \omega \xi d x d t-\int_{0}^{\tau} \int_{\Omega} c\left(\widetilde{v}+G_{0} \zeta\right) \frac{\partial G}{\partial t} \omega \xi d x d t \\
& -\int_{0}^{\tau}(K \nabla G, \nabla \omega) \xi d t \quad \forall \omega \in \mathcal{D}(\Omega), \forall \xi \in \mathcal{D}(0, \tau)
\end{aligned}
$$


and by density of $\mathcal{D}(0, \tau) \otimes \mathcal{D}(\Omega)$ into $L^{2}\left(0, \tau ; H_{0}^{1}(\Omega)\right)$, we obtain

$$
\begin{aligned}
& d \int_{0}^{\tau}\left\langle\frac{\partial^{2} \widetilde{T}}{\partial t^{2}}, w\right\rangle_{H^{-1}(\Omega), H_{0}^{1}(\Omega)} d t+\int_{0}^{\tau} \int_{\Omega} c\left(\widetilde{v}+G_{0} \zeta\right) \frac{\partial \widetilde{T}}{\partial t} w d x d t+\int_{0}^{\tau}(K \nabla \widetilde{T}, \nabla w) d t \\
& =\int_{0}^{\tau} \int_{\Omega}^{\tau} \phi\left(\widetilde{v}+G_{0} \zeta\right) w d x d t-d \int_{0}^{\tau} \int_{\Omega} \frac{\partial^{2} G}{\partial t^{2}} w d x d t-\int_{0}^{\tau} \int_{\Omega} c\left(\widetilde{v}+G_{0} \zeta\right) \frac{\partial G}{\partial t} w d x d t \\
& -\int_{0}^{\tau}(K \nabla G, \nabla w) d t \quad \forall w \in L^{2}\left(0, \tau ; H_{0}^{1}(\Omega)\right) .
\end{aligned}
$$

\section{Numerical simulations}

Motivated by injection moulding $\left({ }^{20-23}\right)$ we consider a model problem with $\Omega=(0, L)^{2}, L=0.2 \mathrm{~m}$, $s=0 \mathrm{~m} / \mathrm{s}, \zeta(t)=1+0.01 t$ for all $t \in[0, \tau]$. The temperature at the boundary of $\Omega$ is given by an increasing function of the time variable on the interval $\left[0, \tau_{g}\right]$ and then a constant temperature $T_{G}$. More precisely

$$
\begin{aligned}
& G(t)=3\left(T_{G}-T_{0}\right)\left(\frac{t}{\tau_{g}}\right)^{4}-8\left(T_{G}-T_{0}\right)\left(\frac{t}{\tau_{g}}\right)^{3}+6\left(T_{G}-T_{0}\right)\left(\frac{t}{\tau_{g}}\right)^{2}+T_{0} \quad \forall t \in\left[0, \tau_{g}\right], \\
& G(t)=T_{G} \quad \forall t \in\left[\tau_{g}, \tau\right] .
\end{aligned}
$$

Hence we may define $G(x, t)=G(t)$ for all $(x, t) \in \Omega \times[0, \tau]$. We consider a velocity parabolic profile for $G_{0}$, namely

$$
G_{0}(x)=\left(4 \frac{x_{2}\left(L-x_{2}\right)}{L^{2}}, 0\right) \quad \forall x=\left(x_{1}, x_{2}\right) \in \Omega .
$$

We assume $f \equiv 0, \phi \equiv 0, v_{0}=G_{0}, T_{0}=300 \mathrm{~K}, T_{1}=0 \mathrm{~K} / \mathrm{s}, T_{G}=350 \mathrm{~K}$ and $\tau_{g}=0.3 \mathrm{~s}$. The temperature dependent viscosity $\mu(T)$ is an affine function, which may be obtained via a linearization of the Arrhenius model leading to

$$
\mu(T)=\mu_{0}\left(1+\alpha\left(T-T_{\text {ref }}\right)\right)
$$

and we consider similarly

$$
c(v)=c_{0}(1+\beta\|v\|)
$$

with $\alpha=-0.03, \beta=0.02, T_{\text {ref }}=325 \mathrm{~K}$. Let us observe that, as long as the temperature $T$ is less than $358 \mathrm{~K}$, the temperature dependent viscosity will satisfy assumption (7). The physical data are given by

$$
\mu_{0}=0.1, \quad \ell=0.015
$$

$\left(\operatorname{see}^{35}\right)$ and

$$
K_{i j}=1 \text { for } i, j \in\{1,2\}, \quad c_{0}=1000 .
$$

Finally we let $d=k * c_{0}$ where $k$ is the thermal relaxation time. We solve the approximate problems with FreeFem++ packages ${ }^{1}$ with $\mathrm{P} 1 \mathrm{~b} / \mathrm{P} 1$ space discretization for problems $\left(\mathrm{P}_{\mathrm{h} \text { flow }}\right)\left({ }^{35,36}\right)$ and $\mathrm{P} 1$ space 

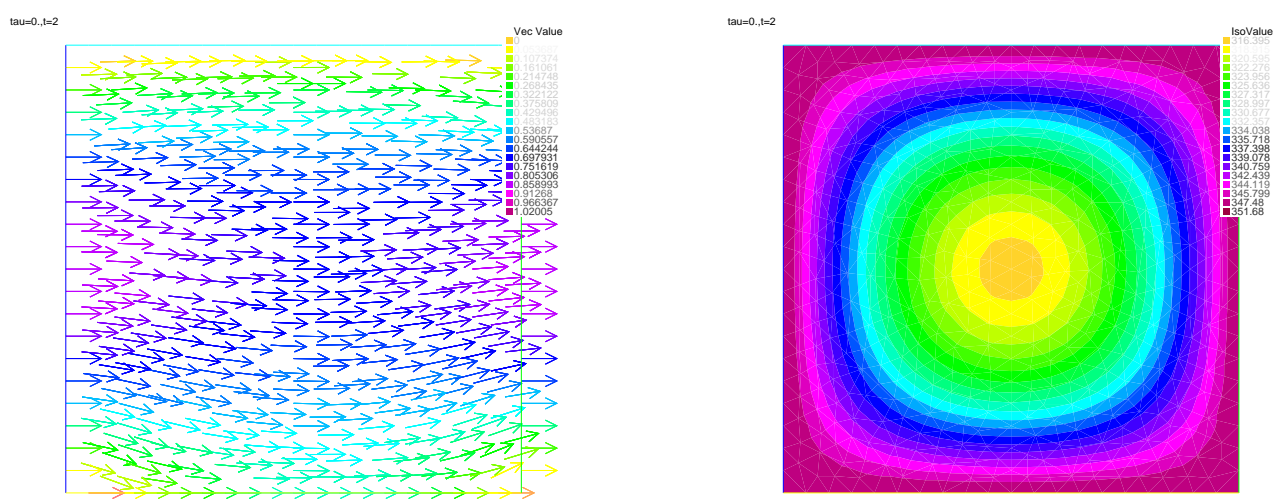

Figure 1. The fluid velocity (left) and temperature (right) with $k=0$ at $t=2 \mathrm{~s}$
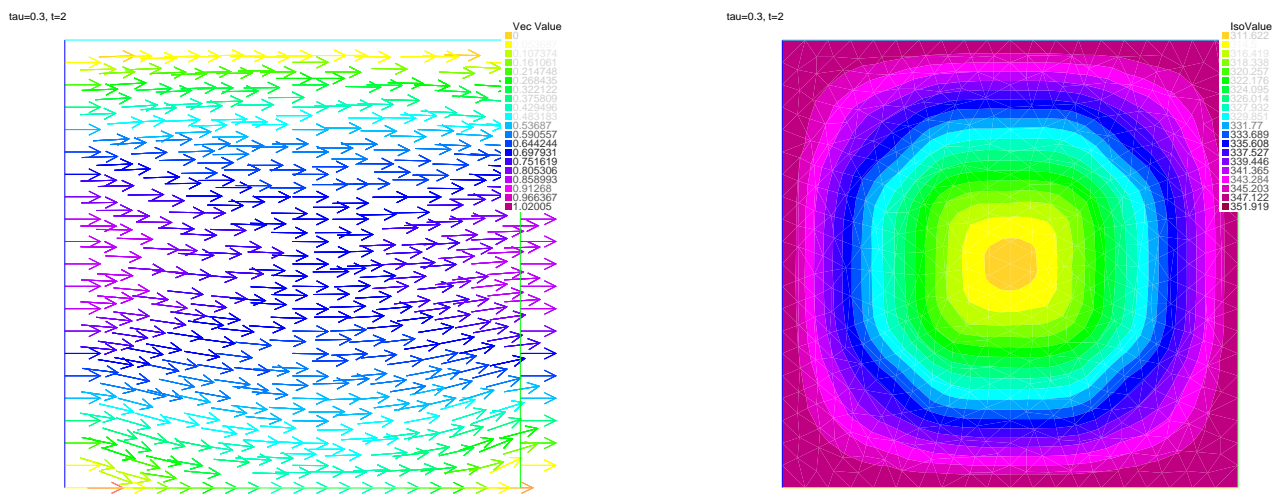

Figure 2. The fluid velocity (left) and temperature (right) with $k=0.3$ at $t=2 \mathrm{~s}$

discretization for problems $\left(\mathrm{P}_{\mathrm{h} \text { heat }}^{\mathrm{n}}\right)$ and 20 space nodes per edge. We consider two different values of $k$, namely $k=0$ (leading to the classical Fourier's heat law) and $k=0.3$ (see ${ }^{37}$ ). The time interval is $[0, \tau]=[0,5]$ and the time-splitting parameter $h=\frac{\tau}{N}$ is chosen such that condition (15) is satisfied. We present in the next figures the temperature and velocity fields at $t=2 \mathrm{~s}$ and $t=5 \mathrm{~s}$.

As expected we can observe slip and non-slip zones along $\Gamma_{0}$ and the influence of the thermal relaxation time on the diffusion of the temperature.

\section{Notes}

1. The authors would like to thank their colleagues A. Agouzal and O. Gipouloux for their advices about the use of FreeFem++ packages. 

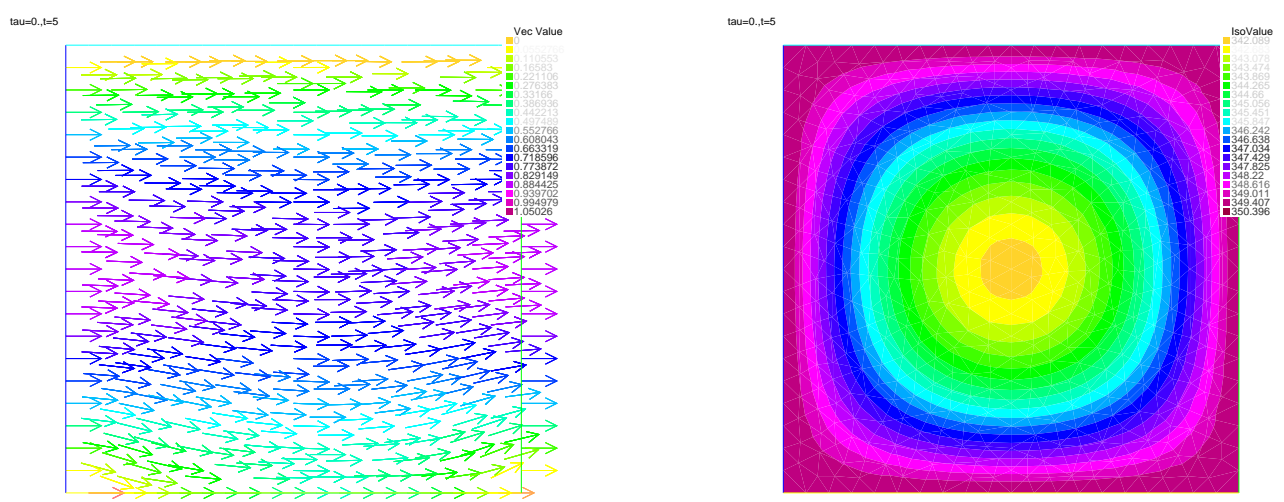

Figure 3. The fluid velocity (left) and temperature (right) with $k=0$ at $t=5 \mathrm{~s}$
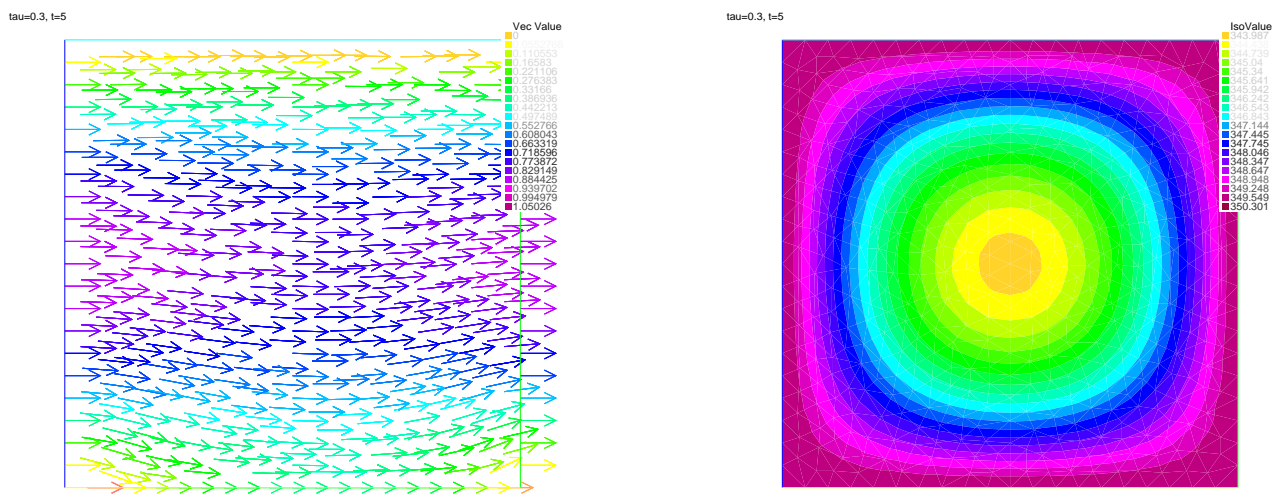

Figure 4. The fluid velocity (left) and temperature (right) with $k=0.3$ at $t=5 \mathrm{~s}$

\section{References}

1. J. Fourier. Théorie analytique de la chaleur, Firmin Didot, Paris, 1822 (New edition Jacques Gabay, Sceaux, 1988).

2. J.C. Maxwell. On the dynamic theory of gases, Phil. Trans. Roy. Soc., 157 (1867) 49-88.

3. L. Onsager. Reciprocal relations in irreversible processes I and II, Phys. Rev, 37 (1931) 405-426 and 38 (1931) 2265-2279.

4. H.E. Wilhelm, S.H. Choi. Nonlinear hyperbolic theory of thermal waves in metal, J. Chemical Physics, 63 (1975) 2119-2123.

5. J.I. Frankel, B. Vick, M.N. Özisik. General formulation and analysis of hyperbolic heat conduction in composite media, Internat J. Heat Mass Trans., 30 (1987) 1293-1305.

6. K. Mitra, S. Kumar, A. Vedavarz et al. Experimental-evidence of hyperbolic heat-conduction in processed meat, J. Heat Transf. - Trans ASME, 117 (1995) 568-573.

7. S. Volz, M. Lallemand, J.B. Saulnier. Analyse de la conduction de la chaleur aux temps ultra-courts dans 
un solide par la thermodynamique irréversible étendue et la dynamique moléculaire, Revue Générale de Thermique, 36(11) (1997) 826-835.

8. W.B. Lor, H.S. Chu. Effects of interface thermal resistance on heat transfer in a composite medium using the thermal wave model, Int. J. Heat Mass Trans., 43 (2000) 653-663.

9. W. Roetzel, N. Putra, S.K. Das. Experiment and analysis for non-Fourier conduction in materials with nonhomogeneous inner structure, Inter. J. Therma Sciences, 42 (2003) 541-522.

10. K.C. Liu. Analysis of dual-phase-lag thermal behaviour in layered films with temperature-dependant interface thermal resistance, J. Phys. D-Appl Phys., 38 (2005) 3722-3732.

11. C.S. Tsai, Y.C. Lin, C.I. Hung. A study on the non-Fourier effects in spherical media due to sudden temperature changes on the surfaces, Heat Mass Trans., 41(46) (2005) 709-716.

12. D.D. Joseph, L. Preziosi. Heat waves, Rev. Modern Physics, 61 (1989) 41-73 and Addendum, Rev. Modern Physics, 61 (1990) 375-391.

13. D.D. Joseph, L. Preziosi. Addendum to the paper "Heat waves", Rev. Modern Physics, 62 (1990) 375-391.

14. D.S. Chandrasekharaiah. Hyperbolic thermoelasticity: a review of recent literature, Appl. Mech. Rev., 51 (1998) 705-729.

15. D. Jou, J. Casas-Vàsquez, G. Lebon. Extended irreversible thermodynamics revisited, Mathematical and Computer Modelling, Rep. Progr. Phys., 62 (1998) 1035-1142.

16. C. Cattaneo. Sulla Conduzione del Calore, Atti. del Seminario Matematico e Fisico Dellà Universita di Modena, 3 (1948) 83-101.

17. C. Cattaneo. On a form of heat equation which eliminates the paradox of instantaneous propagation, C. R. Acad. Sci. Paris, (1958) 431-433.

18. M. Fang, R.P. Gilbert. Non-isothermal, Non-Newtonien Hele Shaw flows within Cattaneo's heat flux law, Mathematical and Computer Modelling, 46 (2007) 765-775.

19. F. White. Vicous fluid flow, Second edition, McGraw-Hill Inc., New-York, 1991.

20. S.C. Chen, Y.C. Chen, N.T. Cheng. Simulation of injection-compression mold-fillig process, Int. Comm. Heat Mass Transfer, 25 (1998) 907-917.

21. S.C. Chen, Y.C. Chen, H.S. Peng. Simulation of Injection-Compression-Molding Process. II. Influence of Process Characteristics on Part Shrinkage, J. Applied Polymer Science, 75 (2000) 1640-1654.

22. J.-F. Hétu, D.M. Gao, A. Garcia-rejon, G. Salloum. 3D Finite Element Method for the Simulation of the Filling Stage in Injection Molding, Polymer Engineering and Science, 38(2) (1998) 223-236.

23. F. Ilinca, J.-F. Hétu. Three-dimensional finite element solution of gas-assisted injection moulding, Int. J. Numer. Meth. Engng, 53(8) (2002) 2003-2017.

24. F. Reif. Fundamentals of statistical and thermal physics, McGraw-Hill Book Company, New-York, 1965.

25. H. Hervet, L. Léger, Flow with slip at the wall: from simple to complex fluids, C. R. Acad. Sci. Paris Physique, 4 (2003) 241-249.

26. M. Boukrouche, R. El Mir. On the Navier-Stokes system in a thin film flow with Tresca free boundary condition and its asymptotic behavior, Bull. Math. Soc. Sc. Math. Roumanie, 48(96-2) (2005) 139-163.

27. M. Boukrouche, F. Saidi. Non isothermal lubrication problem with Tresca fluid-solid interface law, part I, Nonlinear Analysis Real World App., 7(5) (2006) 1145-1166.

28. G. Duvaut, J.L. Lions. Les inéquations en mécanique et physique, Dunod, Gauthiers-Villars, Paris, 1972.

29. M. Boukrouche, I. Boussetouan, L. Paoli. Global existence for 3D Navier-Stokes flows with Tresca's friction boundary conditions, submitted to Quart. App. Math., 2016.

30. M. Boukrouche, I. Boussetouan, L. Paoli. Non-isothermal Navier-Stokes system with mixed boundary conditions 
and friction law: Uniqueness and regularity properties, Nonlinear Analysis T.M.A., 102 (2014) 168-185.

31. M. Boukrouche, I. Boussetouan, L. Paoli. Existence for non-isothermal fluid flows with Tresca's friction and Cattaneo's heat law, J. Math. Anal. Appl., 427(1) (2015) 499-514.

32. J.L. Lions, E. Magenes. Problèmes aux limites non homogènes, Dunod, Paris, 1968.

33. V. Girault, P.A. Raviart. Finite element approximation of the Navier-Stokes equations, Springer-Verlag, Berlin, 1979.

34. J. Simon. Compact sets in the space $L^{p}(0, T ; B)$, Ann. Mat. Pura Applic., 146 (1987) 65-96.

35. M. Ayadi, M.K. Gdoura, T. Sassi. Mixed formulation for Stokes problem with Tresca friction, C. R. Acad. Sci. Paris Série 1, 348 (2010) 1069-1072.

36. M. Ayadi, L. Baffico, M.K. Gdoura, T. Sassi. Error estimates for Stokes problem with Tresca friction conditions, ESAIM Math. Model. Numer. Anal., 48(5) (2014) 1413-1429.

37. F. Ekoue, A. Fouache d'Halloy, D. Gigon, G. Plantamp, E. Zadman. Maxwell-Cattaneo regularization of heat equation, World Acad. Sci. Eng. Tech., 77 (2013) 44-47. 